\title{
Discovery of Highly Potent, Selective, and Orally Efficacious p300/CBP Histone Acetyltransferases Inhibitors
}

Yaxi Yang, ${ }^{\left[\mathrm{a},{ }^{\S]}\right.}$ Rukang Zhang, ${ }^{\left[\mathrm{b},{ }^{\S]}\right.}$ Zhaojun Li, ${ }^{\left[\mathrm{c},{ }^{\S]}\right.}$ Lianghe Mei, ${ }^{[\mathrm{d}]}$ Shili Wan, ${ }^{[\mathrm{a}]}$ Hong Ding, ${ }^{[b]}$ Zhifeng Chen, ${ }^{[b]}$ Jing Xing, ${ }^{[b]}$ Huijin Feng, ${ }^{[a]}$ Jie Han, ${ }^{[b]}$ Hualiang Jiang, ${ }^{[b]}$ Mingyue Zheng, ${ }^{\left[\mathrm{b}, \mathrm{e}^{*}\right]}$ Cheng Luo, ${ }^{\left[\mathrm{b}, \mathrm{e},{ }^{*}\right]}$ and Bing Zhou ${ }^{\left[\mathrm{a}, \mathrm{e}, \mathrm{f}^{*}\right]}$

[a] Department of Medicinal Chemistry, State Key Laboratory of Drug Research, Shanghai Institute of Materia Medica, Chinese Academy of Sciences, 555 Zuchongzhi Road, Shanghai 201203, China. E-mail: zhoubing@simm.ac.cn

[b] Drug Discovery and Design Center, State Key Laboratory of Drug Research, Shanghai Institute of Materia Medica, Chinese Academy of Sciences, 555 Zuchongzhi Road, Shanghai 201203, China. E-mail: cluo@simm.ac.cn; myzheng@simm.ac.cn;

[c] School of Information Management, Dezhou University, No. 566 University Rd. West, Dezhou 253023, Shandong, China

[d] Suzhou Institute of Drug Innovation, Shanghai Institute of Materia Medica, Chinese Academy of Sciences, 108 Yuxin Road, Suzhou, Jiangsu 213000, China

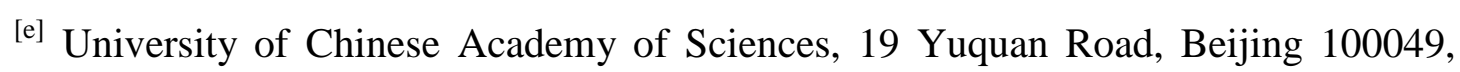
China

[f] Hangzhou Institute for Advanced Study, University of Chinese Academy of Sciences, Hangzhou 310024, China

$\S$ These authors contributed equally. 


\section{Table of contents}

Table S1. Structure and activity of published p300 inhibitors employed for model

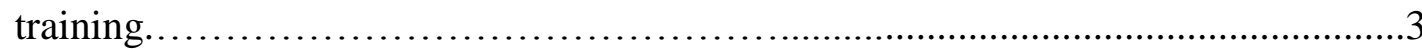

Figure S1. Top 20 potential p300 inhibitors generated by the AI pipeline. .14

Figure S2. The t-SNE projection of random drug-like molecules from ChEMBL, macrocycle molecules, published p300 inhibitors and the top 20 AI proposed p300 inhibitors .15

Figure S3. Illustration of the potential p300 inhibitor generator training process...16

Figure S4. Enrichment evaluation of p300 binding affinity prediction........................................................... 16

Table S2. In vitro permeability measurements in Caco-2 cells....................17

Figure S5. Western blotting result of B026 and A-485 in MV-4-11 cells (24 h)......17

The HPLC, ${ }^{1} \mathrm{H}$ NMR, and ${ }^{13} \mathrm{C}$ NMR Spectra of compound B026...................18 
Table S1. Structure and activity of published p300 inhibitors employed for model training.

\begin{tabular}{|c|c|c|c|c|}
\hline Name & SMILES & $\begin{array}{l}\mathrm{IC}_{50} \\
(\mathrm{nM})\end{array}$ & $\begin{array}{c}\mathrm{Kd} \\
(\mathrm{nM})\end{array}$ & $\begin{array}{l}\text { Delta Tm } \\
\left({ }^{\circ} \mathrm{C}\right)\end{array}$ \\
\hline $\begin{array}{c}\text { CHEMBL109274 } \\
0\end{array}$ & $\begin{array}{c}\mathrm{CC}(=\mathrm{O}) \mathrm{Nc} 1 \mathrm{ccc}(/ \mathrm{N}=\mathrm{N} / \mathrm{c} 2 \mathrm{c}(\mathrm{S}(=\mathrm{O})( \\
=\mathrm{O}) \mathrm{O}) \mathrm{cc} 3 \mathrm{cc}(\mathrm{NC}(=\mathrm{O}) \mathrm{Nc} 4 \mathrm{ccc} 5 \mathrm{c}(\mathrm{O}) \\
\mathrm{c}(/ \mathrm{N}=\mathrm{N} / \mathrm{c} 6 \mathrm{cccc} 6) \mathrm{c}(\mathrm{S}(=\mathrm{O})(=\mathrm{O}) \mathrm{O}) \mathrm{c} \\
\mathrm{c} 5 \mathrm{c} 4) \mathrm{ccc} 3 \mathrm{c} 2 \mathrm{O}) \mathrm{cc} 1\end{array}$ & 13700 & - & - \\
\hline $\begin{array}{c}\text { CHEMBL377478 } \\
7\end{array}$ & $\begin{array}{c}\mathrm{N}=\mathrm{C}(\mathrm{N}) \mathrm{NCCC}[\mathrm{C} @ \mathrm{H}] 1 \mathrm{NC}(=\mathrm{O}) / \mathrm{C} \\
(=\mathrm{C} / \mathrm{c} 2 \mathrm{ccc}(\mathrm{O}) \mathrm{c}(\mathrm{OCC}[\mathrm{C} @ \mathrm{H}](\mathrm{NC}(= \\
\mathrm{O}) \mathrm{C}[\mathrm{C} @ @](\mathrm{O})(\mathrm{CC}(=\mathrm{O}) \mathrm{N}[\mathrm{C} @ \mathrm{H}]( \\
\mathrm{CC}(=\mathrm{O}) \mathrm{O}) \mathrm{C}(=\mathrm{O}) \mathrm{O}) \mathrm{C}(=\mathrm{O}) \mathrm{O}) \mathrm{C}(=\mathrm{O} \\
) \mathrm{O}) \mathrm{c} 2) \mathrm{NC} 1=\mathrm{O}\end{array}$ & 11 & - & - \\
\hline $1 \mathrm{i}$ & $\begin{array}{l}\mathrm{O}=\mathrm{C} 1 / \mathrm{C}(=\mathrm{C} / \mathrm{c} 2 \mathrm{cc}(\mathrm{Br}) \mathrm{c}(\mathrm{O}) \mathrm{c}(\mathrm{Br}) \mathrm{c} 2) \\
\mathrm{CCC} / \mathrm{C} 1=\mathrm{C} / \mathrm{c} 1 \mathrm{cc}(\mathrm{Br}) \mathrm{c}(\mathrm{O}) \mathrm{c}(\mathrm{Br}) \mathrm{c} 1\end{array}$ & 2300 & - & - \\
\hline CHEMBL502089 & $\begin{array}{c}\mathrm{COc} 1 \mathrm{cc}(\mathrm{C}(=\mathrm{O}) \mathrm{C} 2=\mathrm{C} 3 \mathrm{OC}(\mathrm{C})(\mathrm{C})[ \\
\mathrm{C} @ \mathrm{H}](\mathrm{CC}=\mathrm{C}(\mathrm{C}) \mathrm{C}) \mathrm{C}[\mathrm{C} @ @] 34 \\
\mathrm{C}[\mathrm{C} @ @ \mathrm{H}](\mathrm{CC}=\mathrm{C}(\mathrm{C}) \mathrm{C}) \mathrm{C}(\mathrm{C})(\mathrm{C})[ \\
\mathrm{C} @ @](\mathrm{CC}=\mathrm{C}(\mathrm{C}) \mathrm{C})(\mathrm{C} 2=\mathrm{O}) \mathrm{C} 4=\mathrm{O}) \\
\mathrm{ccc} 1 \mathrm{O}\end{array}$ & 5000 & - & - \\
\hline $\begin{array}{c}\text { CHEMBL377578 } \\
7\end{array}$ & $\begin{array}{l}\mathrm{COc} 1 \mathrm{ccc}(\mathrm{C}(=\mathrm{O}) \mathrm{C} 2=\mathrm{C} 3 \mathrm{OC}(\mathrm{C})(\mathrm{C})[ \\
\mathrm{C} @ \mathrm{H}](\mathrm{CC}=\mathrm{C}(\mathrm{C}) \mathrm{C}) \mathrm{C}[\mathrm{C} @] 34 \mathrm{C}[\mathrm{C} \\
@ @ \mathrm{H}](/ \mathrm{C}=\mathrm{ClC}(\mathrm{C}) \mathrm{C}) \mathrm{C}(\mathrm{C})(\mathrm{C})[\mathrm{C} @ \\
](\mathrm{CC}=\mathrm{C}(\mathrm{C}) \mathrm{C})(\mathrm{C} 2=\mathrm{O}) \mathrm{C} 4=\mathrm{O}) \mathrm{cc} 1 \mathrm{O}\end{array}$ & 5000 & - & - \\
\hline $\begin{array}{c}\text { CHEMBL377449 } \\
1\end{array}$ & $\begin{array}{c}\mathrm{N}=\mathrm{C}(\mathrm{N}) \mathrm{NCCC}[\mathrm{C} @ \mathrm{H}] 1 \mathrm{NC}(=\mathrm{O}) / \\
\mathrm{C}(=\mathrm{C} / \mathrm{c} 2 \operatorname{ccc}(\mathrm{O}) \mathrm{c}(\mathrm{OCC}[\mathrm{C} @ \mathrm{H}](\mathrm{N} \\
\mathrm{C}(=\mathrm{O}) \mathrm{C}[\mathrm{C} @](\mathrm{O})(\mathrm{CC}(=\mathrm{O}) \mathrm{O}) \mathrm{C}(=\mathrm{O} \\
) \mathrm{O}) \mathrm{C}(=\mathrm{O}) \mathrm{O}) \mathrm{c} 2) \mathrm{NC} 1=\mathrm{O}\end{array}$ & 22 & - & - \\
\hline CHEMBL500215 & $\operatorname{COc} 1 \operatorname{ccc}(\mathrm{C}(=\mathrm{O}) \mathrm{C} 2=\mathrm{C} 3 \mathrm{OC}(\mathrm{C})(\mathrm{C})[$ & - & 1400 & - \\
\hline
\end{tabular}




\begin{tabular}{|c|c|c|c|c|}
\hline & $\begin{array}{c}\mathrm{C} @ @ \mathrm{H}](\mathrm{CC}=\mathrm{C}(\mathrm{C}) \mathrm{C}) \mathrm{C}[\mathrm{C} @] 34 \mathrm{C}[ \\
\mathrm{C} @ @ \mathrm{H}](\mathrm{CC}=\mathrm{C}(\mathrm{C}) \mathrm{C}) \mathrm{C}(\mathrm{C})(\mathrm{C})[\mathrm{C} @ \\
@](\mathrm{CC}=\mathrm{C}(\mathrm{C}) \mathrm{C})(\mathrm{C} 4) \mathrm{C} 2=\mathrm{O}) \mathrm{cc} 1 \mathrm{O}\end{array}$ & & & \\
\hline CHEMBL502489 & $\begin{array}{c}\mathrm{C}=\mathrm{C}(\mathrm{C})[\mathrm{C} @ \mathrm{H}](\mathrm{CC}=\mathrm{C}(\mathrm{C}) \mathrm{C}) \mathrm{C}[\mathrm{C} \\
@] 12 \mathrm{C}[\mathrm{C} @ @ \mathrm{H}](\mathrm{CC}=\mathrm{C}(\mathrm{C}) \mathrm{C}) \mathrm{C}(\mathrm{C}) \\
(\mathrm{C})[\mathrm{C} @ @](\mathrm{CC}=\mathrm{C}(\mathrm{C}) \mathrm{C})(\mathrm{C}(=\mathrm{O}) \mathrm{C}( \\
\mathrm{C}(=\mathrm{O}) \mathrm{c} 3 \operatorname{ccc}(\mathrm{O}) \mathrm{c}(\mathrm{O}) \mathrm{c} 3)=\mathrm{C} 1 \mathrm{O}) \mathrm{C} 2= \\
\mathrm{O}\end{array}$ & 1700 & 1800 & - \\
\hline CHEMBL458934 & $\begin{array}{c}\mathrm{CC}(\mathrm{C})=\mathrm{CC}[\mathrm{C} @ \mathrm{H}] 1 \mathrm{C}[\mathrm{C} @] 23 \mathrm{C}[\mathrm{C} \\
@ \mathrm{H}](\mathrm{CC}=\mathrm{C}(\mathrm{C}) \mathrm{C}) \mathrm{C}(\mathrm{C})(\mathrm{C})[\mathrm{C} @ @]( \\
\mathrm{CC}=\mathrm{C}(\mathrm{C}) \mathrm{C})(\mathrm{C}(=\mathrm{O}) \mathrm{C}(\mathrm{C}(=\mathrm{O}) \mathrm{c} 4 \mathrm{ccc}( \\
\mathrm{O}) \mathrm{c}(\mathrm{O}) \mathrm{c} 4)=\mathrm{C} 2 \mathrm{OC} 1(\mathrm{C}) \mathrm{C}) \mathrm{C} 3=\mathrm{O}\end{array}$ & - & 1100 & - \\
\hline $\begin{array}{c}\text { CHEMBL377488 } \\
4\end{array}$ & $\begin{array}{c}\mathrm{C}=\mathrm{C}(\mathrm{C})[\mathrm{C} @ @ \mathrm{H}](/ \mathrm{C}=\mathrm{C} / \mathrm{C}(\mathrm{C}) \mathrm{C}) \mathrm{C}[ \\
\mathrm{C} @] 12 \mathrm{C}[\mathrm{C} @ \mathrm{H}](/ \mathrm{C}=\mathrm{ClC}(\mathrm{C}) \mathrm{C}) \mathrm{C}(\mathrm{C} \\
)(\mathrm{C})[\mathrm{C} @ @](\mathrm{CC}=\mathrm{C}(\mathrm{C}) \mathrm{C})(\mathrm{C}(=\mathrm{O}) \mathrm{C}( \\
\mathrm{C}(=\mathrm{O}) \mathrm{c} 3 \operatorname{ccc}(\mathrm{O}) \mathrm{c}(\mathrm{O}) \mathrm{c} 3)=\mathrm{C} 1 \mathrm{O}) \mathrm{C} 2= \\
\mathrm{O}\end{array}$ & 7000 & - & - \\
\hline 23 & $\begin{array}{c}\mathrm{CNC}(=\mathrm{O}) \mathrm{Nc} 1 \mathrm{ccc} 2 \mathrm{c}(\mathrm{c} 1) \mathrm{CC}[\mathrm{C} @ @] \\
21 \mathrm{OC}(=\mathrm{O}) \mathrm{N}(\mathrm{CC}(=\mathrm{O}) \mathrm{N}(\mathrm{Cc} 2 \mathrm{ccc}(\mathrm{F}) \\
\mathrm{cc} 2)[\mathrm{C} @ @ \mathrm{H}](\mathrm{C} 2 \mathrm{CC} 2) \mathrm{C}(\mathrm{F})(\mathrm{F}) \mathrm{F}) \mathrm{C} \\
1=\mathrm{O}\end{array}$ & 32 & - & - \\
\hline $1 d$ & $\begin{array}{c}\mathrm{O}=\mathrm{C} 1 / \mathrm{C}(=\mathrm{C} / \mathrm{c} 2 \operatorname{ccc}(\mathrm{O}) \mathrm{c}(\mathrm{I}) \mathrm{c} 2) \mathrm{CCC} / \\
\mathrm{C} 1=\mathrm{C} 1 \mathrm{c} 1 \operatorname{ccc}(\mathrm{O}) \mathrm{c}(\mathrm{I}) \mathrm{c} 1\end{array}$ & 8100 & - & - \\
\hline $\begin{array}{c}\text { CHEMBL217118 } \\
9\end{array}$ & $\begin{array}{c}\mathrm{O}=\mathrm{C}(\mathrm{c} 1 \mathrm{ccc}(\mathrm{O}) \mathrm{c}(\mathrm{NC}(=\mathrm{O}) \mathrm{c} 2 \mathrm{cccc} 3 \mathrm{c} \\
\operatorname{ccc} 23) \mathrm{c} 1) \mathrm{c} 1 \mathrm{ccc}(\mathrm{O}) \mathrm{c}(\mathrm{NC}(=\mathrm{O}) \mathrm{c} 2 \mathrm{cc} \\
\operatorname{cc} 3 \operatorname{ccc} c 23) \mathrm{c} 1\end{array}$ & 15200 & - & - \\
\hline 24 & $\begin{array}{l}\mathrm{CNC}(=\mathrm{O}) \mathrm{Nc} 1 \mathrm{ccc} 2 \mathrm{c}(\mathrm{c} 1) \mathrm{CC}[\mathrm{C} @ @] \\
21 \mathrm{OC}(=\mathrm{O}) \mathrm{N}(\mathrm{CC}(=\mathrm{O}) \mathrm{N}(\mathrm{Cc} 2 \mathrm{ccc}(\mathrm{F}) \\
\mathrm{cc} 2)[\mathrm{C} @ @ \mathrm{H}](\mathrm{C}) \mathrm{C}(\mathrm{F})(\mathrm{F}) \mathrm{F}) \mathrm{C} 1=\mathrm{O}\end{array}$ & 60 & - & - \\
\hline
\end{tabular}




\begin{tabular}{|c|c|c|c|c|}
\hline 18 & $\begin{array}{c}\mathrm{C}[\mathrm{C} @ \mathrm{H}](\mathrm{C} 1 \mathrm{CC} 1) \mathrm{N}(\mathrm{Cc} 1 \mathrm{ccccc} 1) \mathrm{C}( \\
=\mathrm{O}) \mathrm{CN} 1 \mathrm{C}(=\mathrm{O}) \mathrm{N}[\mathrm{C} @] 2(\mathrm{CCc} 3 \mathrm{cc}( \\
\mathrm{NS}(\mathrm{C})(=\mathrm{O})=\mathrm{O}) \operatorname{ccc} 32) \mathrm{C} 1=\mathrm{O}\end{array}$ & 16000 & - & - \\
\hline $\begin{array}{c}\text { CHEMBL362237 } \\
3\end{array}$ & $\begin{array}{c}\mathrm{COc} 1 \mathrm{ccc}(\mathrm{CCc} 2 \mathrm{nc} 3 \mathrm{cc}(-\mathrm{c} 4 \mathrm{c}(\mathrm{C}) \mathrm{noc} 4 \\
\mathrm{C}) \mathrm{ccc} 3 \mathrm{n} 2 \mathrm{C}[\mathrm{C} @ \mathrm{H}](\mathrm{C}) \mathrm{N} 2 \mathrm{CCOC} \\
\mathrm{C} 2) \mathrm{cc} 1 \mathrm{Cl}\end{array}$ & - & 32 & 10.4 \\
\hline 22 & $\begin{array}{c}\mathrm{CNC}(=\mathrm{O}) \mathrm{Nc} 1 \mathrm{ccc} 2 \mathrm{c}(\mathrm{c} 1) \mathrm{CC}[\mathrm{C} @ @] \\
21 \mathrm{OC}(=\mathrm{O}) \mathrm{N}(\mathrm{CC}(=\mathrm{O}) \mathrm{N}(\mathrm{Cc} 2 \mathrm{ccccc} 2 \\
)[\mathrm{C} @ \mathrm{H}](\mathrm{C}) \mathrm{C} 2 \mathrm{CC} 2) \mathrm{C} 1=\mathrm{O}\end{array}$ & 25 & - & - \\
\hline 21 & $\begin{array}{c}\mathrm{CNC}(=\mathrm{O}) \mathrm{Nc} 1 \mathrm{ccc} 2 \mathrm{c}(\mathrm{c} 1) \mathrm{CC}[\mathrm{C} @ @] \\
21 \mathrm{OC}(=\mathrm{O}) \mathrm{N}(\mathrm{CC}(=\mathrm{O}) \mathrm{N}(\mathrm{Cc} 2 \mathrm{ccccc} 2 \\
)[\mathrm{C} @ \mathrm{H}](\mathrm{C}) \mathrm{C} 2 \mathrm{CC} 2) \mathrm{C} 1=\mathrm{O}\end{array}$ & 121 & - & - \\
\hline 17 & $\begin{array}{c}\mathrm{COC}(=\mathrm{O}) \mathrm{Nc} 1 \mathrm{ccc} 2 \mathrm{c}(\mathrm{c} 1) \mathrm{CC}[\mathrm{C} @ @] \\
21 \mathrm{NC}(=\mathrm{O}) \mathrm{N}(\mathrm{CC}(=\mathrm{O}) \mathrm{N}(\mathrm{Cc} 2 \mathrm{ccccc} 2 \\
)[\mathrm{C} @ \mathrm{H}](\mathrm{C}) \mathrm{C} 2 \mathrm{CC} 2) \mathrm{C} 1=\mathrm{O}\end{array}$ & 1000 & - & - \\
\hline 20 & $\begin{array}{c}\mathrm{CNC}(=\mathrm{O}) \mathrm{Nc} 1 \mathrm{ccc} 2 \mathrm{c}(\mathrm{c} 1) \mathrm{CC}[\mathrm{C} @ @] \\
21 \mathrm{NC}(=\mathrm{O}) \mathrm{N}(\mathrm{CC}(=\mathrm{O}) \mathrm{N}(\mathrm{Cc} 2 \mathrm{ccccc} 2 \\
)[\mathrm{C} @ \mathrm{H}](\mathrm{C}) \mathrm{C} 2 \mathrm{CC} 2) \mathrm{C} 1=\mathrm{O}\end{array}$ & 170 & - & - \\
\hline 19 & $\begin{array}{c}\mathrm{CNC}(=\mathrm{O}) \mathrm{Nc} 1 \mathrm{ccc} 2 \mathrm{c}(\mathrm{c} 1) \mathrm{CC}[\mathrm{C} @ @] \\
21 \mathrm{NC}(=\mathrm{O}) \mathrm{N}(\mathrm{CC}(=\mathrm{O}) \mathrm{N}(\mathrm{Cc} 2 \mathrm{ccccc} 2 \\
)[\mathrm{C} @ \mathrm{H}](\mathrm{C}) \mathrm{C} 2 \mathrm{CC} 2) \mathrm{C} 1=\mathrm{O}\end{array}$ & 240 & - & - \\
\hline $\begin{array}{c}\text { CHEMBL379997 } \\
7\end{array}$ & $\begin{array}{c}\mathrm{C} \# \mathrm{CCNC}(=\mathrm{O}) \mathrm{c} 1 \mathrm{ccc}(\mathrm{N} 2 \mathrm{~N}=\mathrm{C}(\mathrm{C}) / \mathrm{C} \\
(=\mathrm{C} / \mathrm{c} 3 \mathrm{ccc}(-\mathrm{c} 4 \mathrm{cc}(\mathrm{C}) \mathrm{c}(\mathrm{C}) \mathrm{cc} 4[\mathrm{~N}+](= \\
\mathrm{O})[\mathrm{O}-]) \mathrm{o} 3) \mathrm{C} 2=\mathrm{O}) \mathrm{cc} 1\end{array}$ & 14100 & - & - \\
\hline $\begin{array}{c}\text { CHEMBL341653 } \\
6\end{array}$ & $\begin{array}{c}\mathrm{O}=\mathrm{C}(\mathrm{O}) \mathrm{CCSc} 1 \mathrm{cc}(\mathrm{NS}(=\mathrm{O})(=\mathrm{O}) \mathrm{c} 2 \mathrm{c} \\
\operatorname{cc}(\mathrm{Br}) \mathrm{cc} 2) \mathrm{c} 2 \mathrm{cccc} 2 \mathrm{c} 1 \mathrm{O}\end{array}$ & 1000 & - & - \\
\hline $\begin{array}{c}\text { CHEMBL323588 } \\
7\end{array}$ & $\begin{array}{c}\mathrm{O}=\mathrm{S}(=\mathrm{O})(\mathrm{Nc} 1 \mathrm{cc}(\mathrm{Sc} 2 \mathrm{nc}[\mathrm{nH}] \mathrm{n} 2) \mathrm{c}( \\
\mathrm{O}) \mathrm{c} 2 \mathrm{cccc} 12) \mathrm{c} 1 \mathrm{ccc}(\mathrm{Br}) \mathrm{cc} 1\end{array}$ & 1100 & - & - \\
\hline CHEMBL323589 & $\mathrm{O}=\mathrm{S}(=\mathrm{O})(\mathrm{Nc} 1 \mathrm{cc}(\mathrm{Sc} 2 \mathrm{nnnn} 2-\mathrm{c} 2 \mathrm{cccc}$ & 3100 & - & - \\
\hline
\end{tabular}




\begin{tabular}{|c|c|c|c|c|}
\hline 0 & $\mathrm{c} 2) \mathrm{c}(\mathrm{O}) \mathrm{c} 2 \mathrm{cccc} 12) \mathrm{c} 1 \mathrm{cccc} 1$ & & & \\
\hline 16 & $\begin{array}{c}\mathrm{CC}(=\mathrm{O}) \mathrm{Nc} 1 \mathrm{ccc} 2 \mathrm{c}(\mathrm{c} 1) \mathrm{CCC} 21 \mathrm{NC}( \\
=\mathrm{O}) \mathrm{N}(\mathrm{CC}(=\mathrm{O}) \mathrm{N}(\mathrm{Cc} 2 \operatorname{ccc} \mathrm{C} 2) \mathrm{C}(\mathrm{C}) \\
\mathrm{C} 2 \mathrm{CC} 2) \mathrm{C} 1=\mathrm{O}\end{array}$ & 780 & - & - \\
\hline $\begin{array}{c}\text { CHEMBL342196 } \\
0\end{array}$ & $\begin{array}{c}\mathrm{CC} 1=\mathrm{NN}(\mathrm{c} 2 \operatorname{ccc}(\mathrm{C}(=\mathrm{O}) \mathrm{O}) \mathrm{cc} 2) \mathrm{C}(= \\
\mathrm{O}) / \mathrm{C} 1=\mathrm{ClCc} 1 \operatorname{ccc}(\mathrm{Cc} 2 \mathrm{cc}(\mathrm{C}) \mathrm{c}(\mathrm{C}) \mathrm{cc} \\
2[\mathrm{~N}+](=\mathrm{O})[\mathrm{O}-]) \mathrm{o} 1\end{array}$ & 1600 & - & - \\
\hline $\begin{array}{c}\text { CHEMBL377465 } \\
5\end{array}$ & $\begin{array}{c}\mathrm{CCC}(=\mathrm{O}) \mathrm{N} 1 \mathrm{CCOc} 2 \mathrm{c}(\mathrm{cc}(-\mathrm{c} 3 \mathrm{ccc}(\mathrm{O} \\
\mathrm{C}) \mathrm{c}(\mathrm{OC}) \mathrm{c} 3) \mathrm{cc} 2 \mathrm{OC}[\mathrm{C} @ \mathrm{H}] 2 \mathrm{CCCN}( \\
\mathrm{C}) \mathrm{C} 2) \mathrm{C} 1\end{array}$ & - & 167 & - \\
\hline $\begin{array}{l}\text { CHEMBL133121 } \\
1\end{array}$ & $\begin{array}{c}\mathrm{O}=\mathrm{C}(\mathrm{O}) \mathrm{CSc} 1 \mathrm{cc}(\mathrm{NS}(=\mathrm{O})(=\mathrm{O}) \mathrm{c} 2 \mathrm{cc} \\
\mathrm{c}(\mathrm{Br}) \mathrm{cc} 2) \mathrm{c} 2 \mathrm{cccc} 2 \mathrm{c} 1 \mathrm{O}\end{array}$ & 330 & - & - \\
\hline $\begin{array}{c}\text { CHEMBL182389 } \\
5\end{array}$ & $\begin{array}{c}\operatorname{COc} 1 \operatorname{ccc}(\mathrm{S}(=\mathrm{O})(=\mathrm{O}) \mathrm{N}(\mathrm{Cc} 2 \operatorname{ccc} 3 \mathrm{c}( \\
\mathrm{c} 2) \mathrm{C}=\mathrm{CC}(\mathrm{C})(\mathrm{C}) \mathrm{O} 3) \mathrm{c} 2 \operatorname{cccc} 2) \mathrm{cc} 1 \\
\text { OC }\end{array}$ & - & 345 & - \\
\hline CHEMBL222328 & $\begin{array}{c}\mathrm{O}=\mathrm{C} 1 / \mathrm{C}(=\mathrm{C} / \mathrm{c} 2 \operatorname{ccc}(\mathrm{O}) \mathrm{c}(\mathrm{Br}) \mathrm{c} 2) \mathrm{CC} \\
\mathrm{C} / \mathrm{C} 1=\mathrm{C} / \mathrm{c} 1 \operatorname{ccc}(\mathrm{O}) \mathrm{c}(\mathrm{Br}) \mathrm{c} 1\end{array}$ & 5000 & - & - \\
\hline $\begin{array}{c}\text { CHEMBL378042 } \\
9\end{array}$ & $\begin{array}{c}\mathrm{CCN} 1 \mathrm{C}(=\mathrm{O}) \mathrm{c} 2 \operatorname{ccc} 3 \mathrm{c}(\mathrm{NS}(=\mathrm{O})(=\mathrm{O} \\
) \mathrm{c} 4 \mathrm{cc}(\mathrm{Br}) \operatorname{ccc} 4 \mathrm{OC}) \operatorname{ccc} 1 \mathrm{c} 23\end{array}$ & - & - & 3.5 \\
\hline 11 & $\begin{array}{c}\mathrm{CC}(\mathrm{C} 1 \mathrm{CC} 1) \mathrm{N}(\mathrm{Cc} 1 \mathrm{cccc} 1) \mathrm{C}(=\mathrm{O}) \mathrm{C} \\
\mathrm{N} 1 \mathrm{C}(=\mathrm{O}) \mathrm{NC} 2(\mathrm{CCc} 3 \operatorname{cc}(\mathrm{C}(\mathrm{N})=\mathrm{O}) \mathrm{c} \\
\operatorname{cc} 32) \mathrm{C} 1=\mathrm{O}\end{array}$ & 4860 & - & - \\
\hline 12 & $\begin{array}{c}\mathrm{CC}(\mathrm{C} 1 \mathrm{CC} 1) \mathrm{N}(\mathrm{Cc} 1 \mathrm{ccccc} 1) \mathrm{C}(=\mathrm{O}) \mathrm{C} \\
\mathrm{N} 1 \mathrm{C}(=\mathrm{O}) \mathrm{NC} 2(\mathrm{CCc} 3 \mathrm{c}(\mathrm{C}(\mathrm{N})=\mathrm{O}) \mathrm{cc} \\
\operatorname{cc} 32) \mathrm{C} 1=\mathrm{O}\end{array}$ & 17900 & - & - \\
\hline 15 & $\begin{array}{c}\mathrm{CC}(\mathrm{C} 1 \mathrm{CC} 1) \mathrm{N}(\mathrm{Cc} 1 \mathrm{cccc} 1) \mathrm{C}(=\mathrm{O}) \mathrm{C} \\
\mathrm{N} 1 \mathrm{C}(=\mathrm{O}) \mathrm{NC} 2(\mathrm{CCc} 3 \mathrm{cc}(\mathrm{C} 4 \mathrm{CC} 4) \mathrm{cc} \\
\mathrm{c} 32) \mathrm{C} 1=\mathrm{O}\end{array}$ & 1710 & - & - \\
\hline CHEMBL342194 & $\operatorname{COc} 1 \operatorname{ccc}(\mathrm{C}=\mathrm{C} 2 \mathrm{C}(=\mathrm{O}) \mathrm{N}(\mathrm{Cc} 3 \operatorname{ccccc}$ & 11400 & - & - \\
\hline
\end{tabular}




\begin{tabular}{|c|c|c|c|c|}
\hline 4 & $\begin{array}{c}\text { 3) } \mathrm{C}(=\mathrm{O}) \mathrm{N}(\mathrm{Cc} 3 \operatorname{cccc} 3) \mathrm{C} 2=\mathrm{O}) \operatorname{cc} 1 \mathrm{O} \\
\mathrm{C}\end{array}$ & & & \\
\hline $\begin{array}{c}\text { CHEMBL203028 } \\
1\end{array}$ & $\begin{array}{c}\mathrm{O}=\mathrm{S}(=\mathrm{O})(\mathrm{Nc} 1 \mathrm{cc}(\mathrm{Sc} 2 \mathrm{ncn}[\mathrm{nH}] 2) \mathrm{c}( \\
\mathrm{O}) \mathrm{c} 2 \mathrm{ccccc} 12) \mathrm{c} 1 \mathrm{ccc} 2 \operatorname{ccc} c 2 \mathrm{c} 1\end{array}$ & 510 & - & - \\
\hline 13 & $\begin{array}{c}\mathrm{COc} 1 \operatorname{ccc} 2 \mathrm{c}(\mathrm{c} 1) \mathrm{CCC} 21 \mathrm{NC}(=\mathrm{O}) \mathrm{N}( \\
\mathrm{CC}(=\mathrm{O}) \mathrm{N}(\mathrm{Cc} 2 \operatorname{cccc} 2) \mathrm{C}(\mathrm{C}) \mathrm{C} 2 \mathrm{CC} 2 \\
) \mathrm{C} 1=\mathrm{O}\end{array}$ & 1600 & - & - \\
\hline 14 & $\begin{array}{c}\mathrm{CC}(\mathrm{C} 1 \mathrm{CC} 1) \mathrm{N}(\mathrm{Cc} 1 \operatorname{ccccc} 1) \mathrm{C}(=\mathrm{O}) \mathrm{C} \\
\mathrm{N} 1 \mathrm{C}(=\mathrm{O}) \mathrm{NC} 2(\mathrm{CCc} 3 \operatorname{cc}(\mathrm{CO}) \operatorname{ccc} 32) \\
\mathrm{C} 1=\mathrm{O}\end{array}$ & 14000 & - & - \\
\hline $\begin{array}{l}\text { CHEMBL380963 } \\
6\end{array}$ & $\begin{array}{l}\mathrm{CCCOc} 1 \mathrm{ccc} 2 \mathrm{c}(\mathrm{c} 1) \mathrm{c}(-\mathrm{c} 1 \mathrm{c}(\mathrm{C}) \mathrm{n}(\mathrm{C}(= \\
\mathrm{O}) \mathrm{c} 3 \operatorname{ccc}(\mathrm{OC}) \mathrm{cc} 3)[\mathrm{nH}] \mathrm{c} 1=\mathrm{O}) \mathrm{cn} 2 \mathrm{C}( \\
\mathrm{C})=\mathrm{O}\end{array}$ & - & - & 2.7 \\
\hline $\begin{array}{l}\text { CHEMBL341652 } \\
5\end{array}$ & $\begin{array}{c}\mathrm{O}=\mathrm{C} 1 \mathrm{CC}(\mathrm{Sc} 2 \mathrm{nnc}(-\mathrm{c} 3 \operatorname{cccc} 3 \mathrm{O}) \mathrm{o} 2) \\
\mathrm{C}(=\mathrm{O}) \mathrm{N} 1 \mathrm{c} 1 \mathrm{ccc}(\mathrm{Br}) \mathrm{cc} 1\end{array}$ & 3700 & - & - \\
\hline $\begin{array}{c}\text { CHEMBL373482 } \\
3\end{array}$ & $\begin{array}{c}\mathrm{CC} 1=\mathrm{NN}(\mathrm{c} 2 \operatorname{ccc}(\mathrm{C}(=\mathrm{O}) \mathrm{O}) \mathrm{cc} 2) \mathrm{C}(= \\
\mathrm{O}) / \mathrm{C} 1=\mathrm{C} / \mathrm{c} 1 \operatorname{ccc}(-\mathrm{c} 2 \mathrm{cc}(\mathrm{C}) \mathrm{c}(\mathrm{C}) \mathrm{cc} 2[ \\
\mathrm{N}+](=\mathrm{O})[\mathrm{O}-]) \mathrm{o} 1\end{array}$ & 320 & - & - \\
\hline $\begin{array}{c}\text { CHEMBL179793 } \\
6\end{array}$ & $\begin{array}{c}\mathrm{CC} 1=\mathrm{NN}(\mathrm{c} 2 \operatorname{ccc}(\mathrm{C}(=\mathrm{O}) \mathrm{O}) \mathrm{cc} 2) \mathrm{C}(= \\
\mathrm{O}) / \mathrm{C} 1=\mathrm{Clc} 1 \operatorname{ccc}(-\mathrm{c} 2 \mathrm{cc}(\mathrm{C}) \mathrm{c}(\mathrm{C}) \mathrm{cc} 2[ \\
\mathrm{N}+](=\mathrm{O})[\mathrm{O}-]) \mathrm{o} 1\end{array}$ & 6800 & - & - \\
\hline 1 & $\begin{array}{c}\mathrm{CC}(\mathrm{C} 1 \mathrm{CC} 1) \mathrm{N}(\mathrm{Cc} 1 \mathrm{ccccc} 1) \mathrm{C}(=\mathrm{O}) \mathrm{C} \\
\mathrm{N} 1 \mathrm{C}(=\mathrm{O}) \mathrm{NC}(\mathrm{Cc} 2 \mathrm{c}[\mathrm{nH}] \mathrm{c} 3 \operatorname{cccc} 23) \\
\mathrm{C} 1=\mathrm{O}\end{array}$ & 5100 & - & - \\
\hline 9 & $\begin{array}{c}\mathrm{CC}(\mathrm{C} 1 \mathrm{CC} 1) \mathrm{N}(\mathrm{Cc} 1 \operatorname{cccc} 1) \mathrm{C}(=\mathrm{O}) \mathrm{C} \\
\mathrm{N} 1 \mathrm{C}(=\mathrm{O}) \mathrm{NC} 2(\mathrm{CCc} 3 \operatorname{cc}(\mathrm{C \# N}) \operatorname{ccc} 32 \\
) \mathrm{C} 1=\mathrm{O}\end{array}$ & 2500 & - & - \\
\hline $\begin{array}{c}\text { CHEMBL342194 } \\
3\end{array}$ & $\begin{array}{l}\operatorname{COc} 1 \operatorname{cc}(\mathrm{C}=\mathrm{C} 2 \mathrm{C}(=\mathrm{O}) \mathrm{N}(\mathrm{Cc} 3 \operatorname{ccccc} 3 \\
\mathrm{C}(=\mathrm{O}) \mathrm{N}(\mathrm{Cc} 3 \operatorname{cccc} 3) \mathrm{C} 2=\mathrm{O}) \operatorname{ccc} 1 \mathrm{O}\end{array}$ & 1500 & - & - \\
\hline
\end{tabular}




\begin{tabular}{|c|c|c|c|c|}
\hline $\begin{array}{c}\text { CHEMBL342194 } \\
2\end{array}$ & $\begin{array}{l}\operatorname{COc} 1 \operatorname{ccc}(\mathrm{C}=\mathrm{C} 2 \mathrm{C}(=\mathrm{O}) \mathrm{N}(\mathrm{Cc} 3 \operatorname{ccccc} \\
3) \mathrm{C}(=\mathrm{O}) \mathrm{N}(\mathrm{Cc} 3 \operatorname{cccc} 3) \mathrm{C} 2=\mathrm{O}) \operatorname{cc} 1 \mathrm{O}\end{array}$ & 5900 & - & - \\
\hline $\begin{array}{c}\text { CHEMBL377032 } \\
2\end{array}$ & $\begin{array}{l}\operatorname{CCOc} 1 \operatorname{ccc}(\mathrm{C}(\mathrm{C})=\mathrm{O}) \operatorname{cc} 1 \mathrm{NC}(=\mathrm{O}) \mathrm{c} 1 \\
\operatorname{cc}(\mathrm{COC} 2 \mathrm{CCCCO} 2) \operatorname{cc}(\mathrm{C}(=\mathrm{O}) \mathrm{O}) \mathrm{c} 1\end{array}$ & - & - & 5.9 \\
\hline $\begin{array}{c}\text { CHEMBL342196 } \\
1\end{array}$ & $\begin{array}{c}\mathrm{Cc} 1 \mathrm{cc}(\mathrm{O}) \operatorname{cc}(\mathrm{C}) \mathrm{c} 1 \mathrm{C}=\mathrm{C} 1 \mathrm{C}(=\mathrm{O}) \mathrm{N}(\mathrm{C} \\
\mathrm{c} 2 \operatorname{cccc} 2) \mathrm{C}(=\mathrm{O}) \mathrm{N}(\mathrm{Cc} 2 \operatorname{cccc} 2) \mathrm{C} 1= \\
\mathrm{O}\end{array}$ & 2900 & - & - \\
\hline $\begin{array}{c}\text { CHEMBL203156 } \\
6\end{array}$ & $\begin{aligned} \mathrm{O}= & \mathrm{C}(\mathrm{O}) \mathrm{CSc} 1 \mathrm{cc}(\mathrm{NS}(=\mathrm{O})(=\mathrm{O}) \mathrm{c} 2 \mathrm{cc} \\
& \mathrm{c}(\mathrm{C}(=\mathrm{O}) \mathrm{O}) \mathrm{cc} 2) \mathrm{c} 2 \mathrm{cccc} 2 \mathrm{c} 1 \mathrm{O}\end{aligned}$ & 660 & - & - \\
\hline $\begin{array}{c}\text { CHEMBL342194 } \\
1\end{array}$ & $\begin{array}{l}\mathrm{O}=\mathrm{C} 1 \mathrm{C}(=\mathrm{Cc} 2 \operatorname{ccc}(\mathrm{O}) \mathrm{c}(\mathrm{O}) \mathrm{c} 2) \mathrm{C}(=\mathrm{O}) \\
\mathrm{N}(\mathrm{Cc} 2 \operatorname{cccc} 2) \mathrm{C}(=\mathrm{O}) \mathrm{N} 1 \mathrm{Cc} 1 \operatorname{ccc} c 1\end{array}$ & 1600 & - & - \\
\hline $\begin{array}{c}\text { CHEMBL342194 } \\
5\end{array}$ & $\begin{array}{l}\operatorname{COc} 1 \operatorname{ccc}(\mathrm{C}=\mathrm{C} 2 \mathrm{C}(=\mathrm{O}) \mathrm{N}(\mathrm{Cc} 3 \operatorname{ccccc} \\
3) \mathrm{C}(=\mathrm{O}) \mathrm{N}(\mathrm{Cc} 3 \operatorname{cccc} 3) \mathrm{C} 2=\mathrm{O}) \operatorname{cc} 1\end{array}$ & 8000 & - & - \\
\hline 5 & $\begin{array}{c}\mathrm{C}[\mathrm{C} @ \mathrm{H}](\mathrm{C} 1 \mathrm{CC} 1) \mathrm{N}(\mathrm{Cc} 1 \mathrm{ccccc} 1) \mathrm{C}( \\
=\mathrm{O}) \mathrm{CN} 1 \mathrm{C}(=\mathrm{O}) \mathrm{N}[\mathrm{C} @ @] 2(\mathrm{CCc} 3 \mathrm{cc} \\
\operatorname{ccc} 32) \mathrm{C} 1=\mathrm{O}\end{array}$ & 510 & - & - \\
\hline 7 & $\begin{array}{c}\mathrm{C}[\mathrm{C} @ \mathrm{H}](\mathrm{C} 1 \mathrm{CC} 1) \mathrm{N}(\mathrm{Cc} 1 \mathrm{ccccc} 1) \mathrm{C}( \\
=\mathrm{O}) \mathrm{CN} 1 \mathrm{C}(=\mathrm{O}) \mathrm{N}[\mathrm{C} @ @] 2(\mathrm{CCc} 3 \mathrm{cc} \\
\operatorname{ccc} 32) \mathrm{C} 1=\mathrm{O}\end{array}$ & 990 & - & - \\
\hline 4 & $\begin{array}{c}\mathrm{CC}(\mathrm{C} 1 \mathrm{CC} 1) \mathrm{N}(\mathrm{Cc} 1 \operatorname{cccc} 1) \mathrm{C}(=\mathrm{O}) \mathrm{C} \\
\mathrm{N} 1 \mathrm{C}(=\mathrm{O}) \mathrm{NC} 2(\mathrm{CCc} 3 \operatorname{ccc} c 32) \mathrm{C} 1= \\
\mathrm{O}\end{array}$ & 1600 & - & - \\
\hline 6 & $\begin{array}{c}\mathrm{C}[\mathrm{C} @ \mathrm{H}](\mathrm{C} 1 \mathrm{CC} 1) \mathrm{N}(\mathrm{Cc} 1 \mathrm{ccccc} 1) \mathrm{C}( \\
=\mathrm{O}) \mathrm{CN} 1 \mathrm{C}(=\mathrm{O}) \mathrm{N}[\mathrm{C} @ @] 2(\mathrm{CCc} 3 \mathrm{cc} \\
\operatorname{ccc} 32) \mathrm{C} 1=\mathrm{O}\end{array}$ & 5060 & - & - \\
\hline 8 & $\begin{array}{c}\mathrm{C}[\mathrm{C} @ \mathrm{H}](\mathrm{C} 1 \mathrm{CC} 1) \mathrm{N}(\mathrm{Cc} 1 \mathrm{ccccc} 1) \mathrm{C}( \\
=\mathrm{O}) \mathrm{CN} 1 \mathrm{C}(=\mathrm{O}) \mathrm{N}[\mathrm{C} @ @] 2(\mathrm{CCc} 3 \mathrm{cc} \\
\operatorname{ccc} 32) \mathrm{C} 1=\mathrm{O}\end{array}$ & 13000 & - & - \\
\hline CHEMBL341652 & $\operatorname{COc} 1 \mathrm{ccc}(-\mathrm{c} 2 \mathrm{nnnc}(\mathrm{SC} 3 \mathrm{CC}(=\mathrm{O}) \mathrm{N}(\mathrm{c} 4$ & 2800 & - & - \\
\hline
\end{tabular}




\begin{tabular}{|c|c|c|c|c|}
\hline 6 & $\operatorname{ccc}(\mathrm{Cl}) \mathrm{cc} 4) \mathrm{C} 3=\mathrm{O})[\mathrm{nH}] 2) \mathrm{cc} 1$ & & & \\
\hline $\begin{array}{c}\text { CHEMBL341653 } \\
7\end{array}$ & $\begin{array}{c}\mathrm{O}=\mathrm{S}(=\mathrm{O})(\mathrm{Nc} 1 \mathrm{cc}(\mathrm{Cl}) \mathrm{c}(\mathrm{O}) \mathrm{c} 2 \mathrm{cccc} 1 \\
2) \mathrm{c} 1 \mathrm{ccc}(\mathrm{Br}) \mathrm{cc} 1\end{array}$ & 1800 & - & - \\
\hline $\begin{array}{c}\text { CHEMBL342194 } \\
0\end{array}$ & $\begin{array}{c}\mathrm{O}=\mathrm{C} 1 \mathrm{C}(=\mathrm{Cc} 2 \operatorname{ccc}(\mathrm{O}) \operatorname{cc} 2) \mathrm{C}(=\mathrm{O}) \mathrm{N}( \\
\mathrm{Cc} 2 \operatorname{cccc} 2) \mathrm{C}(=\mathrm{O}) \mathrm{N} 1 \mathrm{Cc} 1 \mathrm{cccc} 1\end{array}$ & 2100 & - & - \\
\hline $\begin{array}{c}\text { CHEMBL342190 } \\
5\end{array}$ & $\begin{array}{c}\mathrm{O}=\mathrm{C}(\mathrm{c} 1 \mathrm{ccccc} 1) \mathrm{C} 1 \mathrm{C}(=\mathrm{O}) \mathrm{N}(\mathrm{Cc} 2 \mathrm{ccc} \\
\operatorname{cc} 2) \mathrm{C}(=\mathrm{O}) \mathrm{N}(\mathrm{Cc} 2 \mathrm{cccc} 2) \mathrm{C} 1=\mathrm{O}\end{array}$ & - & 2400 & - \\
\hline $\begin{array}{l}\text { CHEMBL380915 } \\
8\end{array}$ & $\begin{array}{l}\mathrm{CC}(=\mathrm{O}) \mathrm{n} 1 \mathrm{cc}(-\mathrm{c} 2 \mathrm{c}(\mathrm{C}) \mathrm{n}(\mathrm{S}(\mathrm{C})(=\mathrm{O})= \\
\mathrm{O}) \mathrm{n}(\mathrm{S}(\mathrm{C})(=\mathrm{O})=\mathrm{O}) \mathrm{c} 2=\mathrm{O}) \mathrm{c} 2 \mathrm{cccc} 21\end{array}$ & - & - & 2.7 \\
\hline $\begin{array}{c}\text { CHEMBL341631 } \\
7\end{array}$ & $\begin{array}{c}\mathrm{O}=[\mathrm{N}+]([\mathrm{O}-]) \mathrm{c} 1 \mathrm{cc}(\mathrm{Br}) \mathrm{c} 2 \mathrm{nsnc} 2 \mathrm{c} 1 \mathrm{~S} \\
\mathrm{c} 1 \mathrm{ncnc} 2[\mathrm{nH}] \mathrm{cnc} 12\end{array}$ & 2200 & - & - \\
\hline 2 & $\begin{array}{l}\mathrm{NS}(=\mathrm{O})(=\mathrm{O}) \mathrm{c} 1 \mathrm{ccc}(\mathrm{NC}(=\mathrm{O}) \mathrm{CN} 2 \mathrm{C}( \\
=\mathrm{O}) \mathrm{SC}(=\mathrm{C} 3 \mathrm{CCCCC} 3) \mathrm{C} 2=\mathrm{O}) \mathrm{cc} 1\end{array}$ & 11500 & - & - \\
\hline $\begin{array}{c}\text { CHEMBL313380 } \\
7\end{array}$ & $\begin{array}{c}\mathrm{CCOC}(=\mathrm{O}) \mathrm{Nc} 1 \mathrm{cc}(-\mathrm{c} 2 \mathrm{ccc}(\mathrm{C}) \mathrm{c}(\mathrm{NS}( \\
\mathrm{C})(=\mathrm{O})=\mathrm{O}) \mathrm{c} 2) \mathrm{nn} 2 \mathrm{c}(\mathrm{C}) \mathrm{nnc} 12\end{array}$ & - & - & 5.4 \\
\hline $\begin{array}{c}\text { CHEMBL377096 } \\
9\end{array}$ & $\begin{array}{c}\mathrm{CCOc} 1 \mathrm{ccc}(\mathrm{C}(\mathrm{C})=\mathrm{O}) \operatorname{cc} 1 \mathrm{NC}(=\mathrm{O}) \mathrm{c} 1 \\
\operatorname{cc}(\mathrm{C}(=\mathrm{O}) \mathrm{O}) \operatorname{cc}(-\mathrm{c} 2 \mathrm{ccncc} 2) \mathrm{c} 1\end{array}$ & - & - & 3.9 \\
\hline $\begin{array}{c}\text { CHEMBL216359 } \\
8\end{array}$ & $\begin{array}{c}\operatorname{COc} 1 \mathrm{cc}(\mathrm{CN}(\mathrm{C}(\mathrm{C}) \mathrm{C}) \mathrm{S}(=\mathrm{O})(=\mathrm{O}) \mathrm{c} 2 \\
\operatorname{ccc} n 2) \operatorname{cc} 2 \mathrm{c} 1 \mathrm{OC}(\mathrm{C})(\mathrm{C}) \mathrm{C}=\mathrm{C} 2\end{array}$ & 5000 & - & - \\
\hline $\begin{array}{c}\text { CHEMBL216359 } \\
9\end{array}$ & $\begin{array}{c}\mathrm{CC}(\mathrm{C}) \mathrm{CN}(\mathrm{Cc} 1 \mathrm{cc}(\mathrm{O}) \mathrm{c} 2 \mathrm{c}(\mathrm{c} 1) \mathrm{C}=\mathrm{CC} \\
(\mathrm{C})(\mathrm{C}) \mathrm{O} 2) \mathrm{S}(=\mathrm{O})(=\mathrm{O}) \mathrm{c} 1 \mathrm{ccc} n 1\end{array}$ & 4000 & - & - \\
\hline $\begin{array}{c}\text { CHEMBL216360 } \\
3\end{array}$ & $\begin{array}{c}\mathrm{CC}(\mathrm{C}) \mathrm{CN}(\mathrm{Cc} 1 \mathrm{cc}(\mathrm{O}) \mathrm{c} 2 \mathrm{c}(\mathrm{c} 1) \mathrm{C}=\mathrm{CC} \\
(\mathrm{C})(\mathrm{C}) \mathrm{O} 2) \mathrm{S}(=\mathrm{O})(=\mathrm{O}) \mathrm{c} 1 \mathrm{ccncc} 1\end{array}$ & 4000 & - & - \\
\hline $\begin{array}{c}\text { CHEMBL341655 } \\
2\end{array}$ & $\begin{array}{l}\mathrm{CCN}(\mathrm{CC}) \mathrm{c} 1 \mathrm{ccc} 2 \mathrm{c}(\mathrm{C}) \mathrm{c}(-\mathrm{c} 3 \mathrm{ccc}(\mathrm{N} 4 \\
\mathrm{C}(=\mathrm{O}) \mathrm{C}=\mathrm{CC} 4=\mathrm{O}) \mathrm{cc} 3) \mathrm{c}(=\mathrm{O}) \mathrm{oc} 2 \mathrm{c} 1\end{array}$ & 260 & - & - \\
\hline $\begin{array}{c}\text { CHEMBL216359 } \\
7\end{array}$ & $\begin{array}{c}\mathrm{CC} 1(\mathrm{C}) \mathrm{C}=\mathrm{Cc} 2 \mathrm{cc}(\mathrm{CN}(\mathrm{C} 3 \mathrm{CCCC} 3) \\
\mathrm{S}(=\mathrm{O})(=\mathrm{O}) \mathrm{c} 3 \operatorname{ccc} \mathrm{c} 3) \operatorname{ccc} 2 \mathrm{O} 1\end{array}$ & 4000 & - & - \\
\hline $\begin{array}{c}\text { CHEMBL342193 } \\
0\end{array}$ & $\begin{aligned} \mathrm{O}= & \mathrm{C} 1 \mathrm{C}(\mathrm{Cc} 2 \operatorname{cccc} 2) \mathrm{C}(=\mathrm{O}) \mathrm{N}(\mathrm{Cc} 2 \mathrm{c} \\
& \operatorname{ccc} 2) \mathrm{C}(=\mathrm{O}) \mathrm{N} 1 \mathrm{Cc} 1 \operatorname{cccc} 1\end{aligned}$ & - & 110 & - \\
\hline
\end{tabular}




\begin{tabular}{|c|c|c|c|c|}
\hline $\begin{array}{c}\text { CHEMBL378050 } \\
8\end{array}$ & $\begin{array}{c}\operatorname{CCN} 1 \mathrm{C}(=\mathrm{O}) \operatorname{c} 2 \operatorname{ccc} 3 \mathrm{c}(\mathrm{S}(=\mathrm{O})(=\mathrm{O}) \\
\mathrm{Nc} 4 \operatorname{cccc} 4 \mathrm{C}(=\mathrm{O}) \mathrm{O}) \operatorname{ccc} 1 \mathrm{c} 23\end{array}$ & - & - & 6.4 \\
\hline $\begin{array}{c}\text { CHEMBL377059 } \\
8\end{array}$ & $\begin{array}{c}\mathrm{CCOc} 1 \operatorname{ccc}(\mathrm{C}(\mathrm{C})=\mathrm{O}) \operatorname{cc} 1 \mathrm{NC}(=\mathrm{O}) \mathrm{c} 1 \\
\operatorname{cc}(\mathrm{C}(=\mathrm{O}) \mathrm{O}) \operatorname{cc}(-\mathrm{c} 2 \cos 2) \mathrm{c} 1\end{array}$ & - & - & 5.9 \\
\hline $\begin{array}{c}\text { CHEMBL216360 } \\
2\end{array}$ & $\begin{array}{c}\mathrm{CC}(\mathrm{C}) \mathrm{CN}(\mathrm{Cc} 1 \mathrm{ccc} 2 \mathrm{c}(\mathrm{c} 1) \mathrm{C}=\mathrm{CC}(\mathrm{C})( \\
\mathrm{C}) \mathrm{O} 2) \mathrm{S}(=\mathrm{O})(=\mathrm{O}) \mathrm{c} 1 \mathrm{nccs} 1\end{array}$ & 5000 & - & - \\
\hline $\begin{array}{c}\text { CHEMBL216360 } \\
1\end{array}$ & $\begin{array}{c}\mathrm{CC}(\mathrm{C}) \mathrm{CN}(\mathrm{Cc} 1 \mathrm{ccc} 2 \mathrm{c}(\mathrm{c} 1) \mathrm{C}=\mathrm{CC}(\mathrm{C})( \\
\mathrm{C}) \mathrm{O} 2) \mathrm{S}(=\mathrm{O})(=\mathrm{O}) \mathrm{c} 1 \mathrm{nccn} 1 \mathrm{C}\end{array}$ & 5000 & - & - \\
\hline $\begin{array}{l}\text { CHEMBL380987 } \\
6\end{array}$ & $\begin{array}{l}\operatorname{COc} 1 \operatorname{ccc}(\mathrm{C}(=\mathrm{O}) \mathrm{n} 2[\mathrm{nH}] \mathrm{c}(=\mathrm{O}) \mathrm{c}(-\mathrm{c} 3 \\
\operatorname{cn}(\mathrm{C}(\mathrm{C})=\mathrm{O}) \mathrm{c} 4 \mathrm{ccccc} 34) \mathrm{c} 2 \mathrm{C}) \mathrm{cc} 1\end{array}$ & - & - & 5.3 \\
\hline $\begin{array}{l}\text { CHEMBL377050 } \\
0\end{array}$ & $\begin{aligned} \mathrm{CC}( & =\mathrm{O}) \mathrm{c} 1 \operatorname{ccc}(\mathrm{OCc} 2 \operatorname{cccc} 2) \mathrm{c}(\mathrm{NC}( \\
& =\mathrm{O}) \mathrm{c} 2 \operatorname{cccc}(\mathrm{C}(=\mathrm{O}) \mathrm{O}) \mathrm{c} 2) \mathrm{c} 1\end{aligned}$ & - & - & 2.6 \\
\hline $\begin{array}{c}\text { CHEMBL378005 } \\
9\end{array}$ & $\begin{array}{c}\mathrm{CCN} 1 \mathrm{C}(=\mathrm{O}) \mathrm{c} 2 \operatorname{ccc} 3 \mathrm{c}(\mathrm{NS}(=\mathrm{O})(=\mathrm{O} \\
) \mathrm{c} 4 \mathrm{ccc}(\mathrm{F}) \operatorname{cc} 4 \mathrm{~F}) \operatorname{ccc} 1 \mathrm{c} 23\end{array}$ & - & - & 4.5 \\
\hline $\begin{array}{l}\text { CHEMBL216360 } \\
0\end{array}$ & $\begin{array}{c}\mathrm{CC}(\mathrm{C}) \mathrm{CN}(\mathrm{Cc} 1 \mathrm{ccc} 2 \mathrm{c}(\mathrm{c} 1) \mathrm{C}=\mathrm{CC}(\mathrm{C})( \\
\mathrm{C}) \mathrm{O} 2) \mathrm{S}(=\mathrm{O})(=\mathrm{O}) \mathrm{c} 1 \mathrm{ccncc} 1\end{array}$ & 4000 & - & - \\
\hline $\begin{array}{c}\text { CHEMBL216359 } \\
4\end{array}$ & $\begin{array}{c}\mathrm{CC}(\mathrm{C}) \mathrm{CN}(\mathrm{Cc} 1 \mathrm{ccc} 2 \mathrm{c}(\mathrm{c} 1) \mathrm{C}=\mathrm{CC}(\mathrm{C})( \\
\mathrm{C}) \mathrm{O} 2) \mathrm{S}(=\mathrm{O})(=\mathrm{O}) \mathrm{c} 1 \mathrm{ccc} n 1\end{array}$ & 5000 & - & - \\
\hline $\begin{array}{l}\text { CHEMBL381475 } \\
2\end{array}$ & $\begin{array}{c}\mathrm{C}[\mathrm{C} @ @ \mathrm{H}] 1 \mathrm{CC}(=\mathrm{O}) \mathrm{Nc} 2 \mathrm{cccc}(-\mathrm{c} 3 \mathrm{c} \\
\mathrm{cc} 4 \mathrm{c}(\mathrm{c} 3) \mathrm{c}(-\mathrm{c} 3 \mathrm{cnn}(\mathrm{C}) \mathrm{c} 3) \mathrm{nn} 4 \mathrm{C}) \mathrm{c} 2 \mathrm{~N} \\
1\end{array}$ & 51 & - & - \\
\hline $\begin{array}{c}\text { CHEMBL341630 } \\
7\end{array}$ & $\begin{array}{c}\mathrm{O}=\mathrm{C}(\mathrm{O}) \mathrm{c} 1 \mathrm{ccc}(-\mathrm{n} 2 \mathrm{nnnc} 2 \mathrm{SC} 2=\mathrm{CS}( \\
=\mathrm{O})(=\mathrm{O}) \mathrm{c} 3 \operatorname{ccc} \mathrm{c} 32) \mathrm{cc} 1\end{array}$ & 7500 & - & - \\
\hline $\begin{array}{l}\text { CHEMBL216359 } \\
5\end{array}$ & $\begin{array}{l}\mathrm{CC} 1(\mathrm{C}) \mathrm{C}=\mathrm{Cc} 2 \mathrm{cc}(\mathrm{CN}(\mathrm{CC} 3 \mathrm{CC} 3) \mathrm{S}( \\
=\mathrm{O})(=\mathrm{O}) \mathrm{c} 3 \operatorname{ccc} \mathrm{cn} 3) \operatorname{ccc} 2 \mathrm{O} 1\end{array}$ & 4000 & - & - \\
\hline $\begin{array}{c}\text { CHEMBL216359 } \\
6\end{array}$ & $\begin{array}{l}\mathrm{CC} 1(\mathrm{C}) \mathrm{C}=\mathrm{Cc} 2 \mathrm{cc}(\mathrm{CN}(\mathrm{C} 3 \mathrm{CCC} 3) \mathrm{S}( \\
=\mathrm{O})(=\mathrm{O}) \mathrm{c} 3 \operatorname{ccc} \mathrm{c} 3) \operatorname{ccc} 2 \mathrm{O} 1\end{array}$ & 4000 & - & - \\
\hline $\begin{array}{c}\text { CHEMBL341632 } \\
1\end{array}$ & $\begin{array}{c}\mathrm{O}=[\mathrm{N}+]([\mathrm{O}-]) \mathrm{c} 1 \mathrm{c}(\mathrm{Sc} 2 \mathrm{nnnn} 2-\mathrm{c} 2 \mathrm{ccc} \\
\mathrm{cc} 2) \mathrm{cc}(\mathrm{Cl}) \mathrm{c} 2 \mathrm{nonc} 12\end{array}$ & 1100 & - & - \\
\hline
\end{tabular}




\begin{tabular}{|c|c|c|c|c|}
\hline $\begin{array}{c}\text { CHEMBL342195 } \\
0\end{array}$ & $\begin{array}{c}\operatorname{COc1cc}(\mathrm{C}=\mathrm{C} 2 \mathrm{C}(=\mathrm{O}) \mathrm{N}(\mathrm{CC}(\mathrm{C}) \mathrm{C}) \mathrm{C} \\
(=\mathrm{O}) \mathrm{N}(\mathrm{CC}(\mathrm{C}) \mathrm{C}) \mathrm{C} 2=\mathrm{O}) \operatorname{ccc} 1 \mathrm{O}\end{array}$ & 4200 & - & - \\
\hline $\begin{array}{c}\text { CHEMBL216359 } \\
2\end{array}$ & $\begin{array}{c}\mathrm{CC}(\mathrm{C}) \mathrm{N}(\mathrm{Cc} 1 \mathrm{ccc} 2 \mathrm{c}(\mathrm{c} 1) \mathrm{C}=\mathrm{CC}(\mathrm{C})(\mathrm{C} \\
) \mathrm{O} 2) \mathrm{S}(=\mathrm{O})(=\mathrm{O}) \mathrm{c} 1 \mathrm{ccc} n 1\end{array}$ & 4000 & - & - \\
\hline $\begin{array}{c}\text { CHEMBL377134 } \\
1\end{array}$ & $\begin{array}{c}\mathrm{CCOc} 1 \mathrm{ccc}(\mathrm{C}(\mathrm{C})=\mathrm{O}) \operatorname{cc} 1 \mathrm{NC}(=\mathrm{O}) \mathrm{c} 1 \\
\operatorname{cc}(\mathrm{COC}) \operatorname{cc}(\mathrm{C}(=\mathrm{O}) \mathrm{O}) \mathrm{c} 1\end{array}$ & - & - & 4.6 \\
\hline $\begin{array}{c}\text { CHEMBL216359 } \\
3\end{array}$ & $\begin{array}{c}\mathrm{CC} 1(\mathrm{C}) \mathrm{C}=\mathrm{Cc} 2 \operatorname{cc}(\mathrm{CN}(\mathrm{C} 3 \mathrm{CC} 3) \mathrm{S}(= \\
\mathrm{O})(=\mathrm{O}) \operatorname{c} 3 \operatorname{ccc} \operatorname{con} 3) \operatorname{ccc} 2 \mathrm{O} 1\end{array}$ & 4000 & - & - \\
\hline $\begin{array}{c}\text { CHEMBL378039 } \\
8\end{array}$ & $\begin{array}{c}\operatorname{CCN} 1 \mathrm{C}(=\mathrm{O}) \operatorname{c} 2 \operatorname{ccc} 3 \mathrm{c}(\mathrm{S}(=\mathrm{O})(=\mathrm{O}) \\
\mathrm{Nc} 4 \operatorname{ccc}(\mathrm{F}) \mathrm{cc} 4) \operatorname{ccc} 1 \mathrm{c} 23\end{array}$ & - & - & 3.3 \\
\hline $\begin{array}{c}\text { CHEMBL378012 } \\
8\end{array}$ & $\begin{array}{c}\mathrm{CCN} 1 \mathrm{C}(=\mathrm{O}) \mathrm{c} 2 \operatorname{ccc} 3 \mathrm{c}(\mathrm{NS}(=\mathrm{O})(=\mathrm{O} \\
) \mathrm{c} 4 \mathrm{ccc}(\mathrm{F}) \mathrm{cc} 4) \operatorname{ccc} 1 \mathrm{c} 23\end{array}$ & - & - & 5.1 \\
\hline $\begin{array}{c}\text { CHEMBL380872 } \\
9\end{array}$ & $\begin{array}{c}\mathrm{CC}(=\mathrm{O}) \mathrm{n} 1 \mathrm{cc}(-\mathrm{c} 2 \mathrm{c}(\mathrm{C}) \mathrm{n}(\mathrm{C}(=\mathrm{O}) \mathrm{N} 3 \mathrm{C} \\
\mathrm{COCC} 3)[\mathrm{nH}] \mathrm{c} 2=\mathrm{O}) \mathrm{c} 2 \mathrm{cccc} 21\end{array}$ & - & - & 2.5 \\
\hline CHEMBL140 & $\begin{array}{c}\operatorname{COc} 1 \mathrm{cc}(/ \mathrm{C}=\mathrm{C} / \mathrm{C}(=\mathrm{O}) \mathrm{CC}(=\mathrm{O}) / \mathrm{C}=\mathrm{C} \\
/ \mathrm{c} 2 \operatorname{ccc}(\mathrm{O}) \mathrm{c}(\mathrm{OC}) \mathrm{c} 2) \operatorname{ccc} 1 \mathrm{O}\end{array}$ & 6500 & - & - \\
\hline CHEMBL116438 & $\begin{array}{c}\mathrm{COc} 1 \mathrm{cc}(/ \mathrm{C}=\mathrm{C} / \mathrm{C}(=\mathrm{O}) / \mathrm{C}=\mathrm{C}(\mathrm{O}) / \mathrm{C}= \\
\mathrm{C} / \mathrm{c} 2 \operatorname{ccc}(\mathrm{O}) \mathrm{c}(\mathrm{OC}) \mathrm{c} 2) \operatorname{ccc} 1 \mathrm{O}\end{array}$ & 6500 & - & - \\
\hline $\begin{array}{c}\text { CHEMBL } 179560 \\
8\end{array}$ & $\begin{array}{c}\mathrm{O}=\mathrm{c} 1 \mathrm{c} 2 \mathrm{cc}([\mathrm{N}+](=\mathrm{O})[\mathrm{O}-]) \mathrm{cnc} 2 \mathrm{sn} 1- \\
\mathrm{c} 1 \mathrm{ccc}(\mathrm{Oc} 2 \mathrm{cccc} 2) \mathrm{cc} 1\end{array}$ & 2430 & - & - \\
\hline $\begin{array}{c}\text { CHEMBL341652 } \\
8\end{array}$ & $\begin{array}{c}\mathrm{Cc} 1 \mathrm{ccc}(\mathrm{N} 2 \mathrm{C}(=\mathrm{O}) \mathrm{CC}(\mathrm{Sc} 3 n n \mathrm{cn} 3-\mathrm{c} 3 \\
\operatorname{ccccc} 3) \mathrm{C} 2=\mathrm{O}) \mathrm{cc} 1\end{array}$ & 9800 & - & - \\
\hline $\begin{array}{l}\text { CHEMBL179561 } \\
3\end{array}$ & $\begin{array}{c}\text { CCCCc1 } 1 \mathrm{cc}(-\mathrm{n} 2 \mathrm{sc} 3 \mathrm{ncc}(-\mathrm{c} 4 \mathrm{cccc} 4) \\
\operatorname{cc} 3 \mathrm{c} 2=\mathrm{O}) \mathrm{cc} 1\end{array}$ & 8280 & - & - \\
\hline $\begin{array}{l}\text { CHEMBL139231 } \\
5\end{array}$ & $\begin{array}{c}\mathrm{O}=\mathrm{c} 1 \mathrm{c} 2 \operatorname{cccc} 2 \operatorname{sn} 1-\mathrm{c} 1 \operatorname{ccc}(\mathrm{S}(=\mathrm{O})(= \\
\mathrm{O}) \mathrm{N} 2 \mathrm{CCCC} 2) \mathrm{cc} 1\end{array}$ & 8030 & - & - \\
\hline $\begin{array}{c}\text { CHEMBL374081 } \\
9\end{array}$ & $\begin{array}{c}\mathrm{CC}(=\mathrm{O}) \mathrm{c} 1 \mathrm{cc}(-\mathrm{c} 2 \mathrm{ccc} 3 \mathrm{nccn} 23) \mathrm{c} 2 \mathrm{cc} \\
(\mathrm{N} 3 \mathrm{CCOCC} 3) \mathrm{ccn} 12\end{array}$ & - & - & 2 \\
\hline CHEMBL342194 & $\mathrm{CC}(\mathrm{C}) \mathrm{CN} 1 \mathrm{C}(=\mathrm{O}) \mathrm{C}(=\mathrm{Cc} 2 \operatorname{ccc}(\mathrm{O}) \mathrm{c}($ & 5400 & - & - \\
\hline
\end{tabular}




\begin{tabular}{|c|c|c|c|c|}
\hline 8 & $\mathrm{O}) \mathrm{c} 2) \mathrm{C}(=\mathrm{O}) \mathrm{N}(\mathrm{CC}(\mathrm{C}) \mathrm{C}) \mathrm{C} 1=\mathrm{O}$ & & & \\
\hline $\begin{array}{c}\text { CHEMBL378147 } \\
1\end{array}$ & $\begin{array}{c}\mathrm{CCN} 1 \mathrm{C}(=\mathrm{O}) \operatorname{c} 2 \operatorname{ccc} 3 \mathrm{c}(\mathrm{S}(=\mathrm{O})(=\mathrm{O}) \\
\mathrm{NC} 4 \mathrm{CCCCC} 4) \operatorname{ccc} 1 \mathrm{c} 23\end{array}$ & - & - & 2.4 \\
\hline $\begin{array}{c}\text { CHEMBL378125 } \\
4\end{array}$ & $\begin{array}{c}\mathrm{CCN} 1 \mathrm{C}(=\mathrm{O}) \mathrm{c} 2 \operatorname{ccc} 3 \mathrm{c}(\mathrm{NS}(=\mathrm{O})(=\mathrm{O} \\
) \mathrm{C} 4 \mathrm{CCCCC} 4) \operatorname{ccc} 1 \mathrm{c} 23\end{array}$ & - & - & 4.7 \\
\hline $\begin{array}{c}\text { CHEMBL377033 } \\
0\end{array}$ & $\begin{array}{c}\mathrm{CCOc} 1 \operatorname{ccc}(\mathrm{C}(\mathrm{C})=\mathrm{O}) \operatorname{cc} 1 \mathrm{NC}(=\mathrm{O}) \mathrm{c} 1 \\
\operatorname{cc}(\mathrm{CO}) \operatorname{cc}(\mathrm{C}(=\mathrm{O}) \mathrm{O}) \mathrm{c} 1\end{array}$ & - & - & 3.7 \\
\hline $\begin{array}{c}\text { CHEMBL376969 } \\
3\end{array}$ & $\begin{array}{c}\mathrm{CC}(=\mathrm{O}) \mathrm{c} 1 \operatorname{ccc}(\mathrm{OCC}(\mathrm{C}) \mathrm{C}) \mathrm{c}(\mathrm{NC}(=\mathrm{O} \\
) \mathrm{c} 2 \operatorname{ccc}(\mathrm{C}(=\mathrm{O}) \mathrm{O}) \mathrm{c} 2) \mathrm{c} 1\end{array}$ & - & - & 3 \\
\hline $\begin{array}{c}\text { CHEMBL376959 } \\
1\end{array}$ & $\begin{array}{c}\mathrm{CC}(=\mathrm{O}) \mathrm{c} 1 \mathrm{ccc}(\mathrm{OCC} 2 \mathrm{CC} 2) \mathrm{c}(\mathrm{NC}(= \\
\mathrm{O}) \mathrm{c} 2 \operatorname{cccc}(\mathrm{C}(=\mathrm{O}) \mathrm{O}) \mathrm{c} 2) \mathrm{c} 1\end{array}$ & - & - & 3.6 \\
\hline $\begin{array}{c}\text { CHEMBL377113 } \\
2\end{array}$ & $\begin{array}{c}\mathrm{CCOc} 1 \operatorname{ccc}(\mathrm{C}(\mathrm{C})=\mathrm{O}) \operatorname{cc} 1 \mathrm{NC}(=\mathrm{O}) \mathrm{c} 1 \\
\operatorname{ccc}(-\mathrm{n} 2 \mathrm{cnnn} 2) \mathrm{c} 1\end{array}$ & - & - & 2.6 \\
\hline CHEMBL33778 & 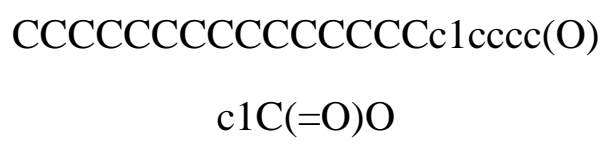 & 4150 & - & - \\
\hline $\begin{array}{c}\text { CHEMBL342194 } \\
7\end{array}$ & $\begin{array}{c}\mathrm{CC}(\mathrm{C}) \mathrm{CN} 1 \mathrm{C}(=\mathrm{O}) \mathrm{C}(=\mathrm{Cc} 2 \operatorname{ccc}(\mathrm{O}) \mathrm{cc} \\
2) \mathrm{C}(=\mathrm{O}) \mathrm{N}(\mathrm{CC}(\mathrm{C}) \mathrm{C}) \mathrm{C} 1=\mathrm{O}\end{array}$ & 8500 & - & - \\
\hline $\begin{array}{l}\text { CHEMBL341631 } \\
6\end{array}$ & $\begin{array}{c}\mathrm{O}=\mathrm{S} 1(=\mathrm{O}) \mathrm{C}=\mathrm{C}(\mathrm{Sc} 2 \mathrm{nnc}(-\mathrm{c} 3 \operatorname{ccncc} 3 \\
) \mathrm{o} 2) \mathrm{c} 2 \operatorname{cccc} 21\end{array}$ & 6200 & - & - \\
\hline CHEMBL455368 & $\begin{array}{c}\mathrm{C}=\mathrm{CC} / \mathrm{C}=\mathrm{C} \backslash \mathrm{C} / \mathrm{C}=\mathrm{C} \backslash \mathrm{CCCCCCCc} 1 \\
\operatorname{cccc}(\mathrm{O}) \mathrm{c} 1 \mathrm{C}(=\mathrm{O}) \mathrm{O}\end{array}$ & 5000 & - & - \\
\hline $\begin{array}{l}\text { CHEMBL341631 } \\
5\end{array}$ & $\begin{array}{c}\mathrm{O}=\mathrm{S} 1(=\mathrm{O}) \mathrm{C}=\mathrm{C}(\mathrm{Sc} 2 \mathrm{nnnn} 2-\mathrm{c} 2 \operatorname{cccc} \mathrm{C} \\
2) \mathrm{c} 2 \operatorname{cccc} 21\end{array}$ & 6200 & - & - \\
\hline $\begin{array}{l}\text { CHEMBL160275 } \\
9\end{array}$ & $\begin{array}{c}\mathrm{O}=\mathrm{S} 1(=\mathrm{O}) \mathrm{C}=\mathrm{C}(\mathrm{Sc} 2 \mathrm{nnc}(-\mathrm{c} 3 \operatorname{ccccc} 3 \\
) \mathrm{o} 2) \mathrm{c} 2 \operatorname{cccc} 21\end{array}$ & 11000 & - & - \\
\hline $\begin{array}{c}\text { CHEMBL377123 } \\
4\end{array}$ & $\begin{array}{c}\mathrm{CCCOc} 1 \operatorname{ccc}(\mathrm{C}(\mathrm{C})=\mathrm{O}) \operatorname{cc} 1 \mathrm{NC}(=\mathrm{O}) \\
\operatorname{c1} \operatorname{cccc}(\mathrm{C}(=\mathrm{O}) \mathrm{O}) \mathrm{c} 1\end{array}$ & - & - & 3.4 \\
\hline $\begin{array}{c}\text { CHEMBL378090 } \\
1\end{array}$ & $\begin{array}{c}\operatorname{CCCCS}(=\mathrm{O})(=\mathrm{O}) \mathrm{Nc} 1 \mathrm{ccc} 2 \mathrm{c} 3 \mathrm{c}(\mathrm{ccc} \\
\mathrm{c} 13) \mathrm{C}(=\mathrm{O}) \mathrm{N} 2 \mathrm{CC}\end{array}$ & - & - & 3.2 \\
\hline
\end{tabular}




\begin{tabular}{|c|c|c|c|c|}
\hline $\begin{array}{c}\text { CHEMBL377015 } \\
0\end{array}$ & $\begin{array}{c}\operatorname{CCOc} 1 \operatorname{ccc}(\mathrm{C}(\mathrm{C})=\mathrm{O}) \operatorname{cc} 1 \mathrm{NC}(=\mathrm{O}) \mathrm{c} 1 \\
\operatorname{cccc}(\mathrm{C}(=\mathrm{O}) \mathrm{O}) \mathrm{c} 1\end{array}$ & - & - & 3.4 \\
\hline $\begin{array}{c}\text { CHEMBL377103 } \\
6\end{array}$ & $\begin{array}{c}\mathrm{CC}(=\mathrm{O}) \mathrm{c} 1 \mathrm{ccc}(\mathrm{C} 2 \mathrm{CC} 2) \mathrm{c}(\mathrm{NC}(=\mathrm{O}) \mathrm{c} \\
2 \operatorname{ccc}(\mathrm{C}(=\mathrm{O}) \mathrm{O}) \mathrm{c} 2) \mathrm{c} 1\end{array}$ & - & - & 3.1 \\
\hline $\begin{array}{c}\text { CHEMBL377567 } \\
1\end{array}$ & $\begin{array}{c}\operatorname{COc} 1 \operatorname{ccc}(\mathrm{S}(=\mathrm{O})(=\mathrm{O}) \mathrm{ON}=\mathrm{C} 2 \mathrm{C}=\mathrm{C}( \\
\mathrm{C}) \mathrm{C}(=\mathrm{O}) \mathrm{C}(\mathrm{C})=\mathrm{C} 2) \mathrm{cc} 1\end{array}$ & 1980 & - & - \\
\hline $\begin{array}{c}\text { CHEMBL179561 } \\
9\end{array}$ & $\begin{array}{c}\mathrm{O}=\mathrm{c} 1 \mathrm{c} 2 \mathrm{cccn} 2 \operatorname{sn} 1 \mathrm{Cc} 1 \mathrm{ccc}(\mathrm{Cl}) \mathrm{c}(\mathrm{Cl}) \\
\mathrm{c} 1\end{array}$ & 5320 & - & - \\
\hline $\begin{array}{c}\text { CHEMBL179562 } \\
4\end{array}$ & $\begin{array}{c}\mathrm{O}=\mathrm{c} 1 \mathrm{c} 2 \mathrm{cccnc} 2 \operatorname{sn} 1 \mathrm{Cc} 1 \mathrm{ccc}(\mathrm{C}(\mathrm{F})(\mathrm{F}) \\
\mathrm{F}) \mathrm{cc} 1\end{array}$ & 5920 & - & - \\
\hline $\begin{array}{c}\text { CHEMBL172093 } \\
9\end{array}$ & $\begin{array}{c}\operatorname{CCOC}(=O) \operatorname{c} 1 \operatorname{ccc}(-n 2 \operatorname{sc} 3 \operatorname{cccc} 3 c 2 \\
=O) c c 1\end{array}$ & 12900 & - & - \\
\hline $\begin{array}{c}\text { CHEMBL208778 } \\
9\end{array}$ & $\begin{array}{c}\operatorname{Cc} 1 \mathrm{cc}(\mathrm{C}) \mathrm{c} 2 \mathrm{c}(=\mathrm{O}) \mathrm{n}(\mathrm{C}(=\mathrm{O}) \mathrm{c} 3 \operatorname{cccs} 3 \\
) \operatorname{sc} 2 \mathrm{n} 1\end{array}$ & 6740 & - & - \\
\hline $\begin{array}{l}\text { CHEMBL160011 } \\
4\end{array}$ & $\begin{array}{c}\mathrm{CNc} 1 \mathrm{nc}(-\mathrm{c} 2 \operatorname{cccc} 2)[\mathrm{n}+](-\mathrm{c} 2 \operatorname{ccc}(\mathrm{C}) \\
\mathrm{cc} 2) \mathrm{s} 1\end{array}$ & 2000 & - & - \\
\hline $\begin{array}{c}\text { CHEMBL380983 } \\
4\end{array}$ & $\begin{array}{c}\mathrm{CC}(=\mathrm{O}) \mathrm{n} 1 \mathrm{cc}(-\mathrm{c} 2 \mathrm{c}(\mathrm{C}) \mathrm{nn} 3 \mathrm{c} 2 \mathrm{OCC} 3) \\
\mathrm{c} 2 \operatorname{ccc} \mathrm{c} 21\end{array}$ & - & - & 2.2 \\
\hline $\begin{array}{l}\text { CHEMBL179561 } \\
8\end{array}$ & $\mathrm{O}=\mathrm{c} 1 \mathrm{c} 2 \mathrm{ccc} n \mathrm{c} 2 \mathrm{sn} 1 \mathrm{Cc} 1 \mathrm{ccc}(\mathrm{Cl}) \mathrm{cc} 1$ & 5740 & - & - \\
\hline $\begin{array}{c}\text { CHEMBL138534 } \\
3\end{array}$ & $\begin{array}{c}\operatorname{Cc} 1 \operatorname{ccc}(-\mathrm{c} 2 \mathrm{nc}(\mathrm{N}) \mathrm{s}[\mathrm{n}+] 2-\mathrm{c} 2 \operatorname{cccc} 2) \\
\mathrm{cc} 1\end{array}$ & 1800 & - & - \\
\hline $\begin{array}{l}\text { CHEMBL341633 } \\
5\end{array}$ & $\begin{array}{c}\mathrm{CNc} 1 \mathrm{nc}(-\mathrm{c} 2 \operatorname{cccc} 2)[\mathrm{n}+](-\mathrm{c} 2 \operatorname{ccccc} 2 \\
) \mathrm{s} 1\end{array}$ & 1900 & - & - \\
\hline CHEMBL318367 & $\mathrm{O}=\mathrm{c} 1 \mathrm{c} 2 \mathrm{cccnc} 2 \operatorname{sn} 1-\mathrm{c} 1 \mathrm{ccc}(\mathrm{Cl}) \mathrm{cc} 1$ & 5710 & - & - \\
\hline CHEMBL491092 & $\begin{array}{c}\mathrm{O}=\mathrm{c} 1 \mathrm{c} \operatorname{csn} 1-\mathrm{c} 1 \mathrm{ccc}(\mathrm{N} 2 \mathrm{CCOCC} 2) \mathrm{cc} \\
1\end{array}$ & 9900 & - & - \\
\hline $\begin{array}{l}\text { CHEMBL173369 } \\
5\end{array}$ & $\operatorname{Cc} 1 \csc (-n 2 \operatorname{sc} 3 \operatorname{ccc} c 3 c 2=0) n 1$ & 8140 & - & - \\
\hline
\end{tabular}




\begin{tabular}{|c|c|c|c|c|}
\hline CHEMBL106011 & $\mathrm{O}=\mathrm{c} 1 \mathrm{c} 2 \mathrm{cccn} 2 \mathrm{sn} 1-\mathrm{c} 1 \mathrm{ccc}(\mathrm{F}) \mathrm{cc} 1$ & 5350 & - & - \\
\hline CHEMBL139155 & $\mathrm{O}=\mathrm{c} 1 \mathrm{c} 2 \mathrm{ccccc} 2 \mathrm{sn} 1-\mathrm{c} 1 \mathrm{nccs} 1$ & 9350 & - & - \\
2 & & & & - \\
\hline CHEMBL295316 & $\mathrm{CC} 1=\mathrm{CC}(=\mathrm{O}) \mathrm{c} 2 \mathrm{c}(\mathrm{O}) \mathrm{cccc} 2 \mathrm{C} 1=\mathrm{O}$ & 2000 & 2000 & - \\
\hline CHEMBL499919 & $\mathrm{O}=\mathrm{c} 1 \mathrm{ccsn} 1-\mathrm{c} 1 \mathrm{ccncc} 1$ & 3000 & - & - \\
\hline CHEMBL487498 & $\mathrm{O}=\mathrm{c} 1 \mathrm{ccsn} 1-\mathrm{c} 1 \mathrm{cccnc} 1$ & 14400 & - & - \\
\hline
\end{tabular}
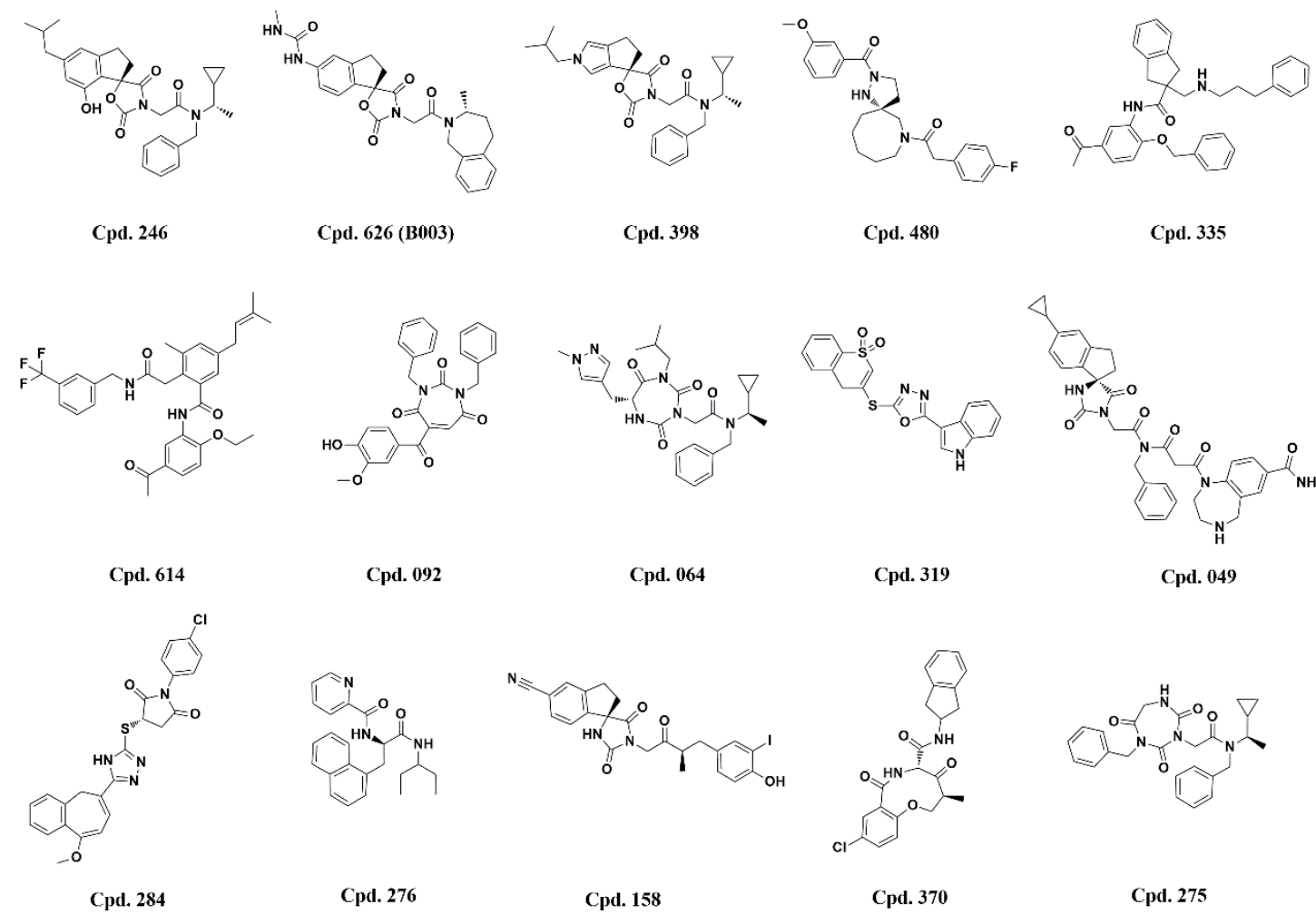

Cpd. 276

Cpd. 158

Cpd. 370

Cpd. 275
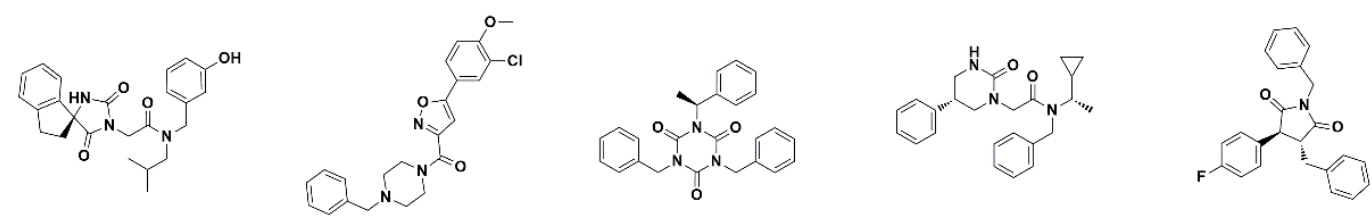

Cpd. 638

Cpd. 554

Cpd. 558

Cpd. 630

Cpd. 297

Figure S1. Top 20 potential P300 inhibitors generated by the AI pipeline. 


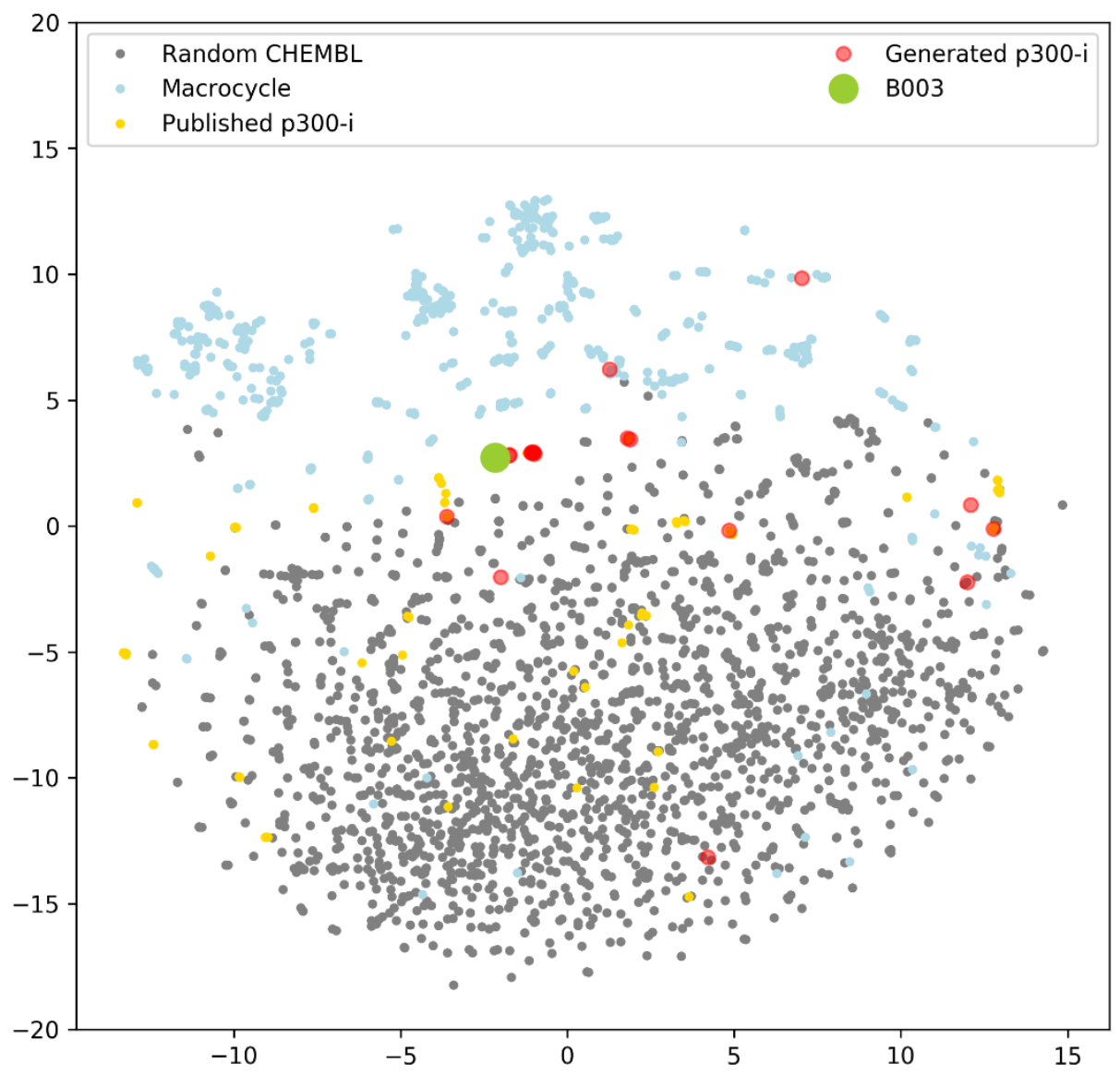

Figure S2. The t-SNE projection of random drug-like molecules from ChEMBL, macrocycle molecules, published p300 inhibitors and the top 20 AI proposed p300 inhibitors. The chemical structure features of each molecule were presented as an ECFP6 512-bit vector. 


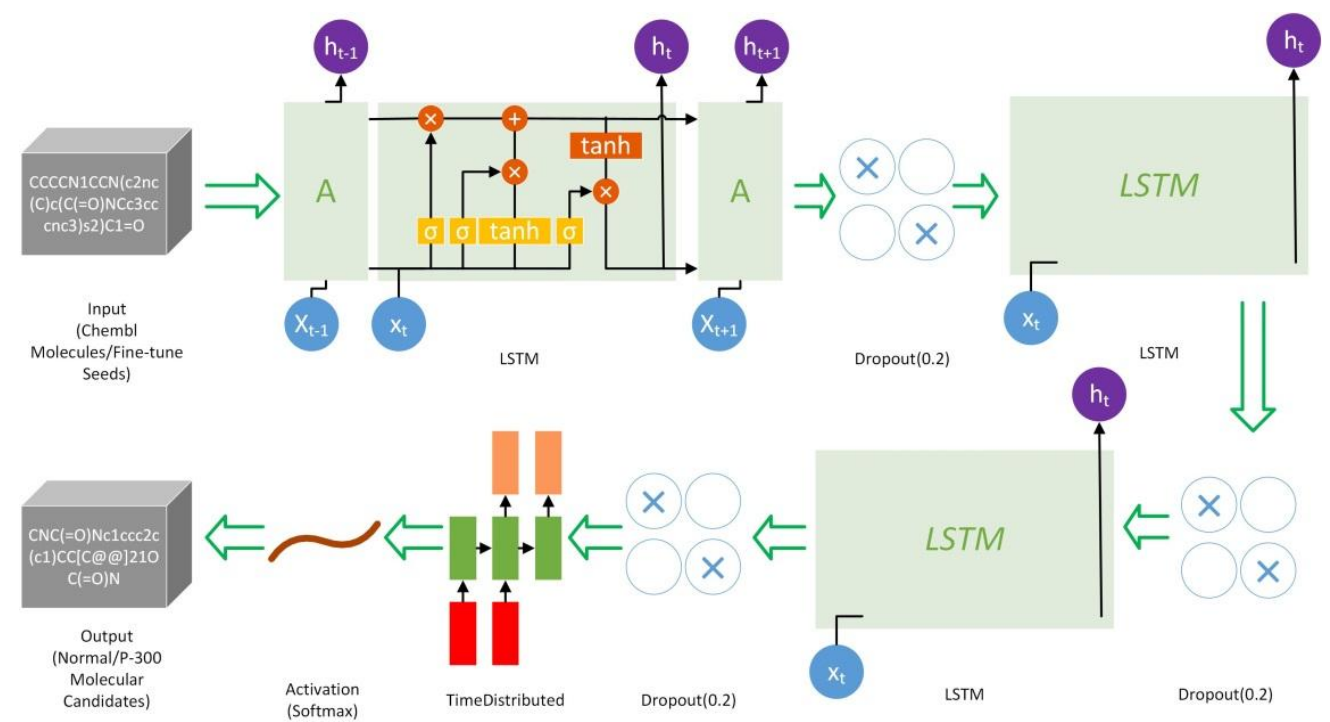

Figure S3. Illustration of the potential p300 inhibitor generator training process.

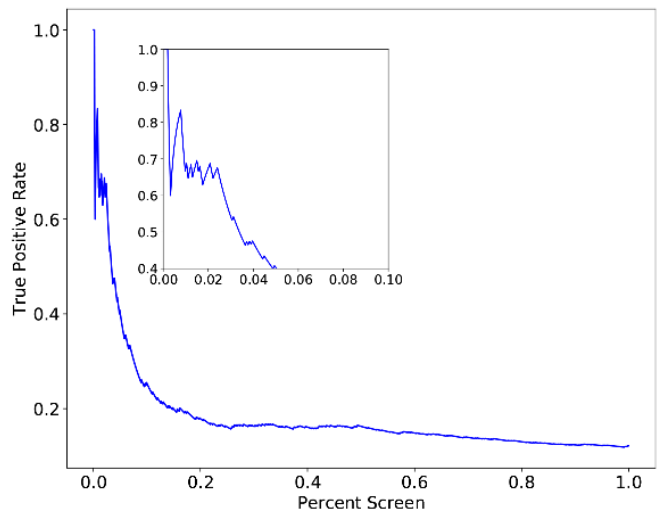

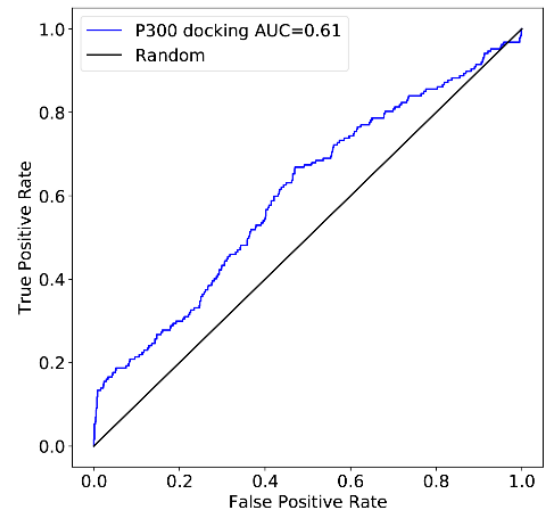

Figure S4. Enrichment evaluation of p300 binding affinity prediction. A, true positive rate against the percentage of screening result. $B$, ROC curve of the docking model. 
Table S2. In vitro permeability measurements in Caco-2 cells.

\begin{tabular}{cccc}
\hline Compound & Flow Direction & Mean Papp $\left(10^{-6} \mathrm{~cm} / \mathrm{s}\right)$ & Efflux ratio \\
\hline \multirow{2}{*}{ B021 } & Apical to Basal & 0.24 & 60 \\
& Basal to Apical & 14.33 & \\
$\mathbf{B 0 2 2}$ & Apical to Basal & 0.26 & 39.59 \\
& Basal to Apical & 10.23 & \\
$\mathbf{B 0 2 3}$ & Apical to Basal & 2.98 & 0.43 \\
& Basal to Apical & 1.29 & 0.31 \\
$\mathbf{B 0 2 4}$ & Apical to Basal & 3.12 & \\
& Basal to Apical & 0.97 & 0.99 \\
\hline
\end{tabular}

\section{$24 \mathrm{~h}$}

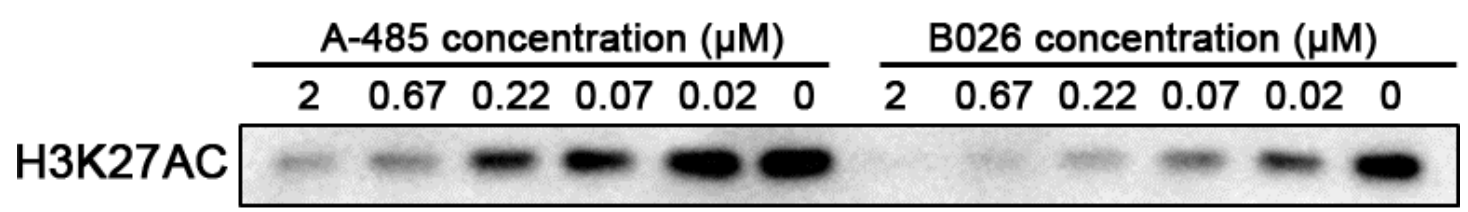

H3

Figure S5. Western blotting result of B026 and A-485 in MV-4-11 cells (24 h). 
The HPLC, ${ }^{1} \mathrm{H}$ NMR, and ${ }^{13} \mathrm{C}$ NMR Spectra of compound B026:

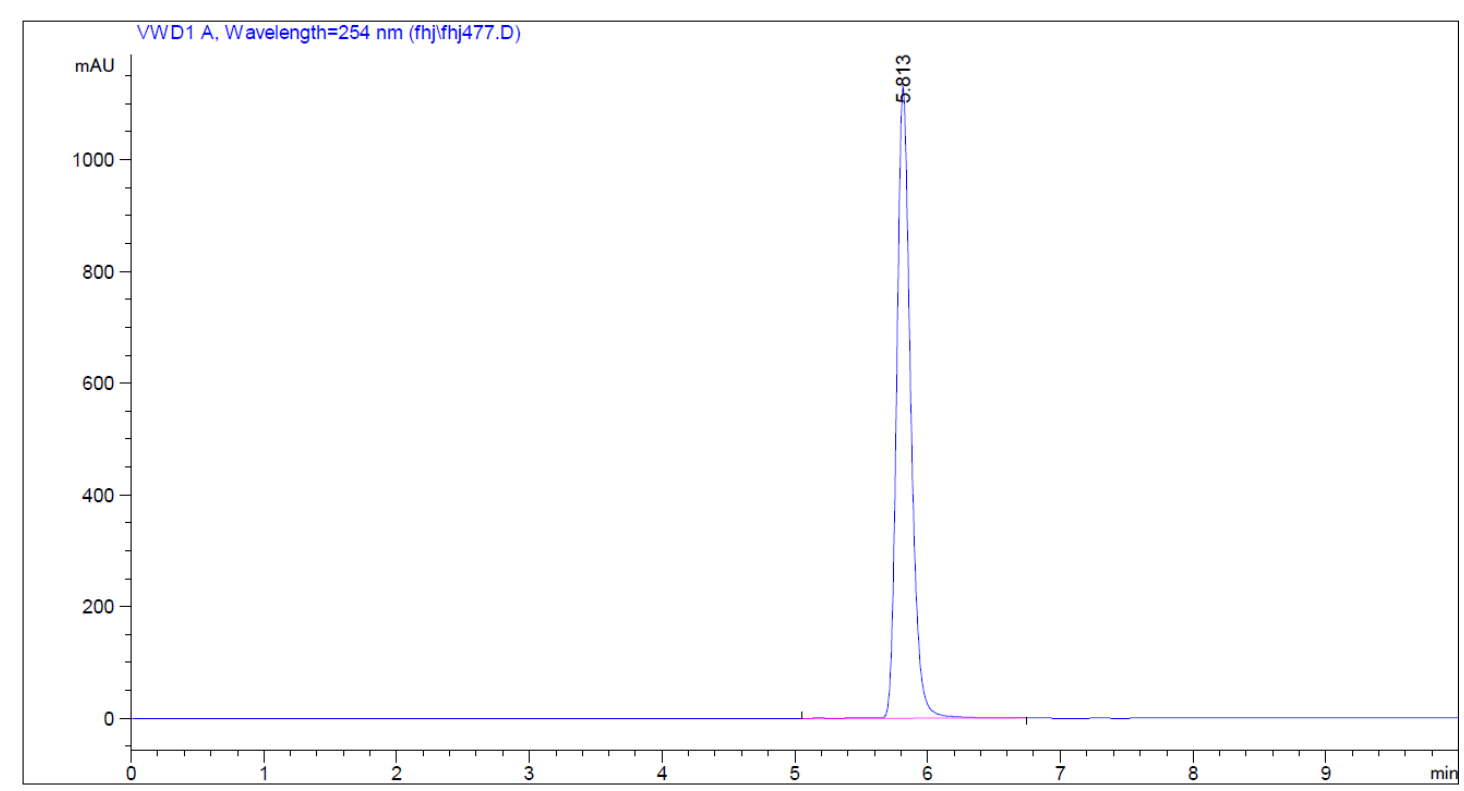

信号 1: VWD1 A, Wavelength=254 nm

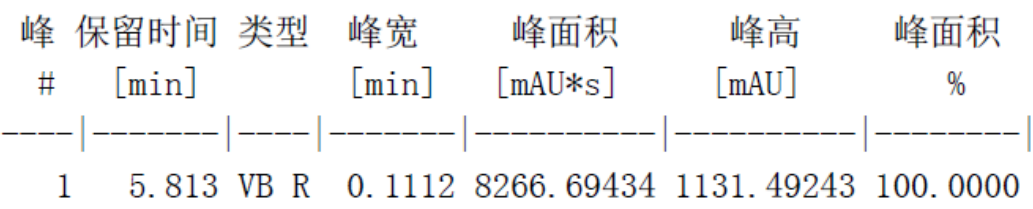




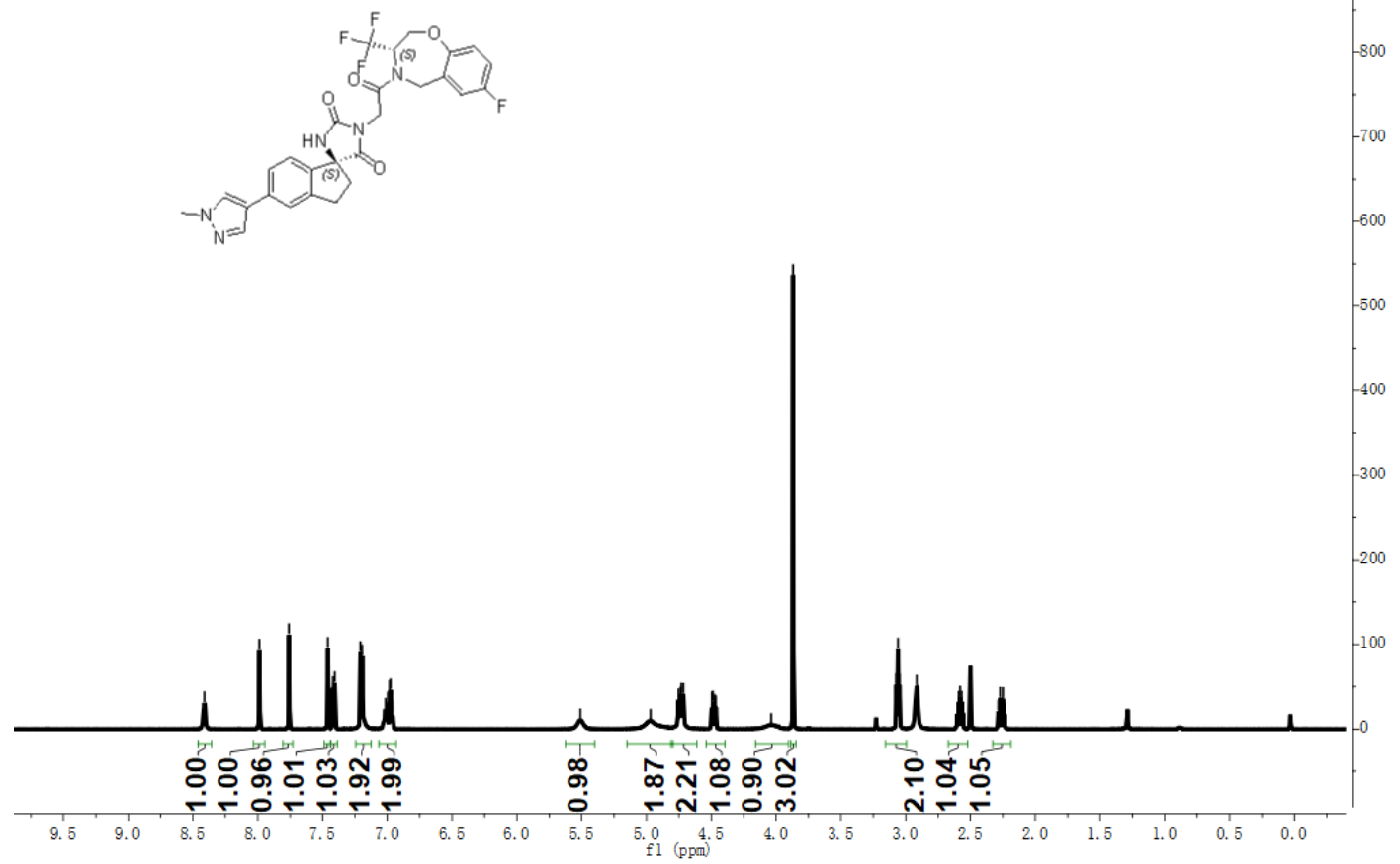

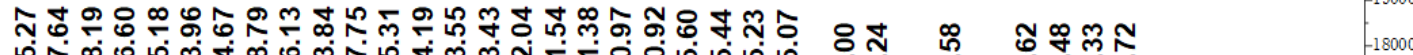

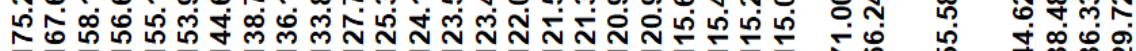

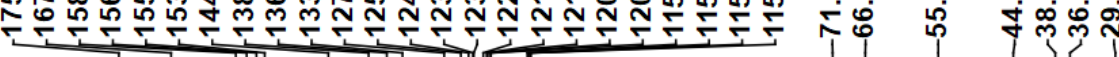

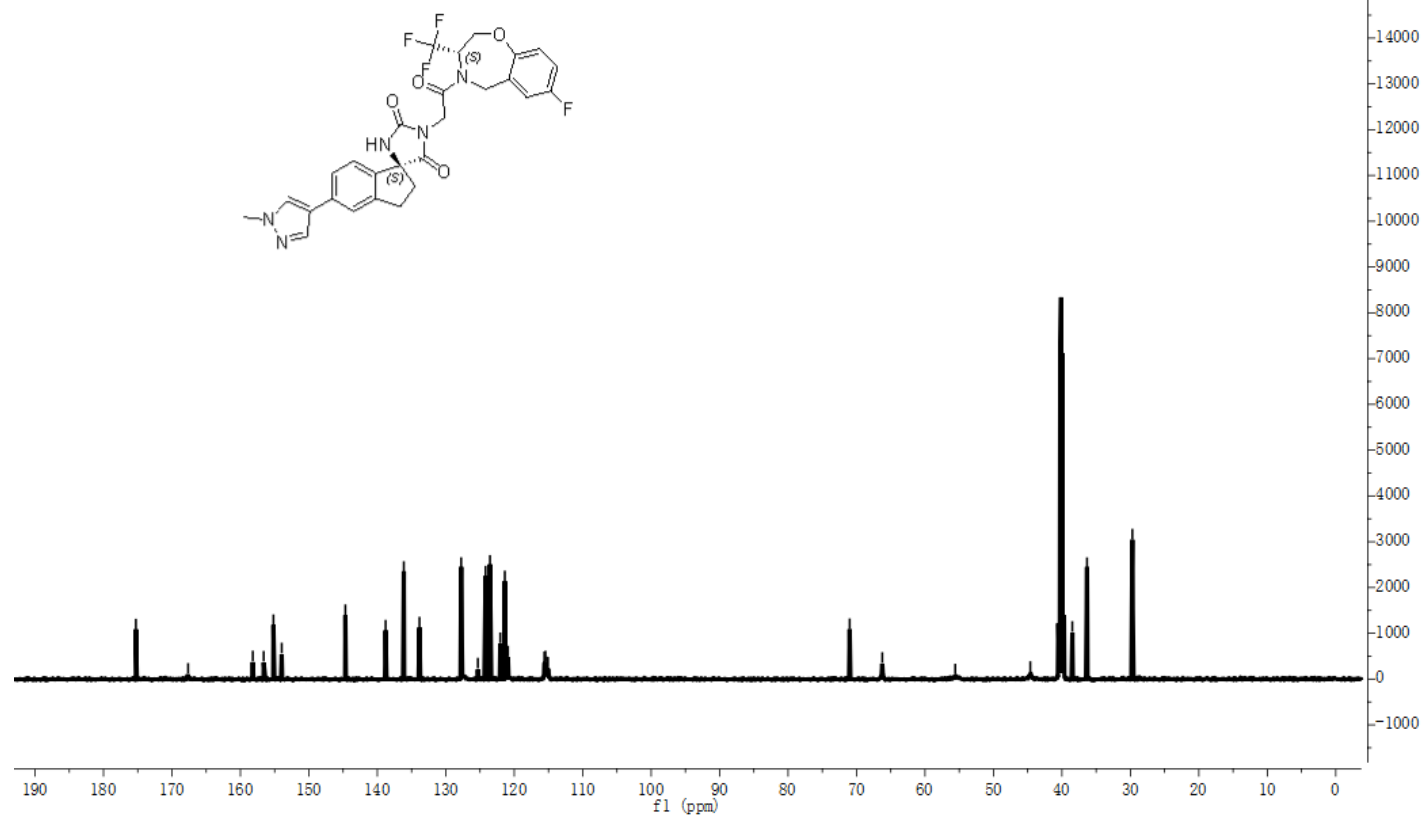

